\title{
What Change in Body Mass Index Is Required to Improve Cardiovascular Outcomes in Childhood and Adolescent Obesity through Lifestyle Interventions: A Meta-Regression
}

\author{
Ahmed Y.M. El-Medany, MSc,' Laura Birch, RD, MSc, Linda P. Hunt, PhD, ${ }^{2}$ \\ Rhys I.B. Matson, MRes, ${ }^{2}$ Amanda H.W. Chong, BSc, ${ }^{2}$ Rhona Beynon, MSc, ${ }^{2}$ \\ Julian Hamilton-Shield, MD, ${ }^{2,3}$ and Rachel Perry, $\mathrm{PhD}^{2}$
}

\section{Abstract}

Background: Using meta-regression, this article aims at establishing the minimum change in BMI-standard deviation score (SDS) needed to improve lipid profiles and blood pressure in children and adolescents with obesity, to aid future trials and guidelines.

Methods: Studies with participants involved in lifestyle interventions, aged 4-19 years, with a diagnosis of obesity according to defined BMI thresholds, were considered for inclusion in a large systematic review. Interventions had to report pre- and postintervention (or mean change in) BMI-SDS, plus either systolic blood pressure (SBP), high-density lipoprotein (HDL) cholesterol, low-density lipoprotein (LDL) cholesterol, and/or triglycerides (TGs). Random-effects meta-regression quantified the relationship between mean change in BMI-SDS and mean change in cardiovascular outcomes.

Results: Seventy-one papers reported various cardiovascular measurements and mean change in BMI-SDS. Fifty-four, 59, 46, and 54 studies were analyzed, reporting a change in SBP, HDL, LDL, and TG, respectively. Reduction in mean BMI-SDS was significantly related to improvements in SBP, LDL, TG, and HDL $(p<0.05)$; BMI-SDS reductions of $1,1.2$, and 0.7 ensured a mean reduction of SBP, LDL, and TG, respectively, although an equivalent value for HDL improvement was indeterminate.

Conclusion: Reductions in mean BMI-SDS of $>1,>1.2$, or $>0.7$ are likely to reduce SBP, LDL, and TG, respectively. Further studies are needed to clarify the optimal duration, intensity, and setting for interventions. Consistency is required regarding derived BMI values to facilitate future systematic reviews and meta-analyses.

Keywords: blood pressure; body mass index-standard deviation score; lifestyle; lipid profile; obesity; pediatrics

\section{Introduction}

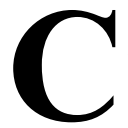
hildhood obesity has reached epidemic proportions, becoming one of the most serious global public health challenges. ${ }^{1}$ In the United Kingdom, around $9.6 \%$ and $20 \%$ of children aged $4-5$ years and $10-11$ years are obese, respectively. ${ }^{2}$ Many of these children have risk factors for hypertension, dyslipidemia, insulin resistance, and adult obesity. 3,4
In children and adolescents, the relationship between cardiovascular disease (CVD) risk factors and early development of atherosclerosis is derived mainly from autopsy studies. ${ }^{5}$ Four large cohort studies, Bogalusa, ${ }^{6-8}$ Muscatine, ${ }^{9,10}$ the Childhood Determinants of Adult Health (CDAH) study, ${ }^{8}$ and the Young Finns Study (YFS), ${ }^{8,11}$ have cited high-density lipoprotein (HDL) cholesterol, low-density lipoprotein (LDL) cholesterol, triglycerides (TGs), and systolic blood pressure (SBP) as

\footnotetext{
'Cardiology Department, Bristol Royal Infirmary, University Hospitals Bristol NHS Foundation Trust, Bristol, United Kingdom.

${ }^{2}$ Level 3 University Hospitals Bristol Education Centre, NIHR Bristol Biomedical Research Centre-Nutrition, Bristol, United Kingdom.

${ }^{3}$ Level 6 University Hospitals Bristol Education Centre, University Hospitals Bristol NHS Foundation Trust, Bristol, United Kingdom.
} 
significant risk factors for adult CVD in children and adolescents with obesity. ${ }^{7,8,10,11}$

In adults, weight loss $\geq 5 \%$ in individuals aged $46 \pm 13$ years has been found to benefit many CVD risk factors ${ }^{12}$ and there are long-established weight reduction guidelines regarding the improvement of adult cardio-metabolic health. ${ }^{13,14}$ There are currently no systematic reviews or meta-analyses assessing the relationship between improvements in BMI-standard deviation score (SDS) and benefits to cardio-metabolic health in children and adolescents; the reduction in BMI-SDS required to improve CVD risk factors such as blood pressure (BP) and lipid profile is unknown.

A 15-year prospective observational study of 1388 overweight children (mean age $11.4 \pm 0.1$ years; $43.8 \%$ male), conducted in a specialized outpatient obesity clinic in north-west Germany, concluded that a BMI-SDS reduction of $\geq 0.25$ significantly improved hypertension, hyper-triglyceridemia, and LDL levels; whereas a BMISDS reduction $\geq 0.5$ doubled this effect. ${ }^{15}$ Importantly, this study lacked an untreated control group, and it therefore did not account for any regression to the mean. Ford et al. reported improvements in insulin sensitivity, lipids, and BP in adolescents with obesity with BMI-SDS reductions of $\geq 0.25$, with greater benefits with reductions of $\geq 0.5$. $^{16}$ Wunsch et al. reported an association between obesity, cardio-metabolic risk factors, and increased carotid intimamedia thickness in children, which was reversible with marked weight loss (BMI-SDS decrease >0.5). ${ }^{17}$

\section{Measuring Change in BMI \\ across Childhood and Adolescence}

BMI, in addition to various other measurements during childhood, is influenced by growth and puberty. Thresholds routinely used in adults cannot be used in pediatrics. Using Cole's Box Cox transformations, measured values of BMI can be standardized into BMI-SDS, in respect to reference populations; these normalize the BMI skewed distribution in childhood. ${ }^{18}$ This provides a normalized measurement for obesity in pediatrics, indicating the degree that an individual BMI lies around the median BMI value. ${ }^{19,20}$

\section{Lifestyle Interventions Affecting \\ Cardio-Metabolic Health in Childhood}

Numerous lifestyle intervention programs are conducted worldwide, with statistically significant reductions in BMISDS. Previous systematic reviews and meta-analyses have assessed the impact of lifestyle interventions on reducing BMI-SDS but have not looked at the reduction in BMISDS required to improve metabolic and cardiovascular parameters.

A Cochrane review by Oude et al., which included 64 randomized controlled trials $(\mathrm{RCTs})(n=5230)$ and focused mainly on dietary, exercise, and behavioral lifestyle interventions, found significant changes in BMI-SDS at 6 months follow-up $(-0.06,95 \%$ confidence interval $[\mathrm{CI}]$ -0.12 to -0.01$)$ in children younger than 12 years (3 RCTs); $(-0.14,95 \% \mathrm{CI}-0.18$ to -0.10$)$ in children 12 years and older ( $3 \mathrm{RCTs}) .{ }^{21}$ A meta-analysis of $33 \mathrm{RCTs}$ by $\mathrm{Ho}$ et al. illustrated a reduction of $-0.10(95 \% \mathrm{CI}-0.18$ to -0.02) in BMI-SDS, with lifestyle interventions versus a "usual care" control group. Interventions included the "traffic light diet" and supervised physical activity sessions. Ho et al. were unable to give definitive recommendations for weight management practice due to the heterogeneity of the included studies. ${ }^{22}$ Another Cochrane review by Colquitt et al. found a mean reduction of -0.3 in BMI-SDS across 4 low-quality evidence RCTs, at 6-12 months follow-up (95\% $\mathrm{CI}-0.4$ to -0.2 ). These trials compared multi-component interventions with usual care. ${ }^{23}$

The complexities and challenges facing obesity interventions are many: behavioral patterns, and the biochemical and physiological nature of the obesity itself may reflect multiple genetic, cultural, and environmental influencers. ${ }^{24}$ Determining a systematic quantification of high-quality evidence, related to the reduction in BMI needed to achieve cardio-metabolic health improvements in the obese pediatric population, would help guide future trials, clinical guidelines, and lifestyle interventions. ${ }^{25-30}$

\section{Study Objectives}

This study aims at establishing the minimum change in BMI-SDS that is necessary to improve lipid profile and BP in children and adolescents with obesity. This article is the third of three papers reporting on the findings from studies identified in a large systematic review. The other two papers focus on changes in percentage body fat, ${ }^{31}$ and inflammation, liver function, and insulin sensitivity measurements - including C-reactive protein, alanine aminotransferase, and the homeostatic model assessment of insulin resistance. ${ }^{32}$

\section{Methods}

This review follows the Preferred Reporting Items for Systematic Reviews and Meta-Analyses (PRISMA) 2010 reporting guideline. ${ }^{33}$ The studies included in this article were identified as a part of large-scale systematic review ( $n=90$ studies; searched up to May 2017), PROSPERO CRD42016025317. This can be accessed via https:// www.crd.york.ac.uk/PROSPERO/. An open-access protocol paper has also been published. ${ }^{34}$

The methodology conducted in this review has been reported in detail in a previous publication. ${ }^{31}$ Completed, published RCTs and nonrandomized studies (cohort studies) of lifestyle interventions, involving participants with obesity aged 4-19 years (as per defined obesity thresholds), were considered for inclusion. This article focuses on lipid profile and BP, particularly SBP, HDL, LDL, and TG. Analyses for diastolic blood pressure (DBP) and total cholesterol (TC) were not included, as SBP, HDL, LDL, and TG were deemed to be more significant predictors of CVD in adulthood based 
on evidence from four large cohort studies. ${ }^{7,8,10,11}$ The TC to HDL cholesterol ratio (TC/HDL), LDL/HDL, very lowdensity lipoproteins (VLDLs), and non-HDL were not included in this article due to the paucity of studies reporting on them ( $n=6$ for TC/HDL, 2 for LDL/HDL, and 1 for VLDL and non-HDL) and the lack of evidence supporting these parameters as predictive of atherosclerotic disease, on transitioning from childhood to adulthood. ${ }^{7,8,10,11}$

Therefore, interventions had to report pre- and postintervention (or mean change in) BMI-SDS (or "z-score"), plus some or all of SBP, HDL, LDL, and/or TG. The BMISDS presented in the studies was used, as opposed to calculating BMI-SDS from a single standard. Appendix A1 summarizes the review's eligibility criteria, data extraction process, and quality assessment.

\section{Quality Assessment}

This study focusses on the association between change in BMI-SDS and change in cardiovascular risk parameters, rather than the specific treatment interventions that effect those changes. Risk of bias tools, such as the Cochrane risk of bias tool, were therefore not used. ${ }^{35}$ The included studies were assessed for methodological quality by two members of the review team during the data extraction process using the Quality Assessment tool utilized in the 2004 Health Technology Assessment (HTA) systematic review of the long-term effects and economic consequences of treatments for obesity and implications for health improvement. ${ }^{36}$ This tool consists of 20 questions, which are combined to give a final score and percentage "quality" rating (Supplementary Data S1-S3 and Supplementary Table S1 for further details). Discrepancies in quality assessment scoring were resolved through discussion.

\section{Analysis}

Data analysis follows the same approach as used in the first paper in this series. ${ }^{31}$ Random-effects meta-regression analyses, as implemented in Stata, ${ }^{37}$ were utilized to quantify the relationships between mean change in BMISDS (independent, predictor variable) and mean change in (1) SBP, (2) LDL, (3) HDL, and (4) TG (target variables), where these target variables were reported.

In contrast to meta-analysis, meta-regression was developed to examine the impact of moderator variables on study effects via a regression-based technique. Here, it was used to study the co-relationships between the predictor variable and target variables across a diverse range of study subsets. The relative effects of the various interventions were not being evaluated, with predictor and target variables instead being considered as correlated "outcomes"; the methodology allows for residual heterogeneity in the target variable not explained by the predictor. ${ }^{38}$ All available subsets documented within each study (i.e., intervention vs. control, boys vs. girls, good vs. poor responders) were included, provided there was no duplication of data.

Subsets were chosen in preference to the total group of participants in each study, if both were reported, to give a greater spread of outcomes. Standard deviations (SDs), as well as means, were required for the changes in the target variables and, if not given explicitly, were calculated from either the standard errors (SEs) or the $95 \%$ CIs of the mean changes. If studies reported only pre- (baseline) and post(intervention or control) values, the SDs of the changes in target variables had to be estimated. The latter required an estimate of the correlation coefficient $(r)$ between pre- and post-results; $r$ was estimated for each subset that reported that all three SDs (pre-, post- and changes) and their median were used.

Where medians and interquartile range (IQR) were reported for baseline and post-intervention results, means and SDs were estimated from them. ${ }^{39,40}$ Where there were missing data, the original authors were contacted, and where missing data remained, attempts were made to estimate these missing values from other results reported in the studies or by imputing possible values using the mean of values from comparable studies. In situations where this was not possible, the study had to be excluded from analysis.

Further exploratory analyses were undertaken by using the percentage of women and the length of the study as additional predictors. Sensitivity analyses using variations of $r$ were carried out in a previous paper in this review but failed to significantly impact the outcomes. ${ }^{31}$

Fitted regression lines are shown together with their 95\% prediction intervals (95\% PI), with individual points representing individual study subgroup results (the mean change in the target variable and the mean change in BMISDS) and the size of the surrounding circles representing the precision of the mean change in the target variable (i.e., the reciprocal of the SE squared). For a given mean change in BMI-SDS, the upper and lower limits of the 95\% PI indicate the range of mean changes in target values that would be expected in future studies. The smallest reduction of mean BMI-SDS associated with improvement in the mean target variable was taken to be the minimum value for which the 95\% PI was wholly less than (or more than) 0 .

\section{Results}

Ninety-eight published articles related to 90 different studies met the inclusion criteria for the systematic review. Figure 1is a flow diagram illustrating the number of papers excluded at each stage of the review. Further information regarding the search results can be found in Supplementary Data S1. The Venn diagram (Supplementary Data S2 and Supplementary Fig. S1) illustrates how many studies were identified for the various markers of metabolic health. Seventy-one studies assessed and reported cardiovascular outcomes.

\section{Outcome Measures}

The BP measures reported included SBP, DBP, 24-hour ambulatory SBP and DBP, and SBP and DBP SDS (SBPSDS and DBP-SDS). Lipid profile measurements reported included HDL, LDL, TC, TG, TC to HDL ratio (TC/HDL), 
LDL to HDL ratio (LDL/HDL), VLDL, and non-HDL cholesterol. All lipid measurements reported (HDL, LDL, and TG) were fasting samples.

For this review, before exclusions (see the Methods section and the Quantitative Analysis section), the BP measures included are SBP ( $n=56$ studies). The lipid profile measures included are HDL $(n=60)$, LDL $(n=47)$, and TG $(n=56)$.

\section{Narrative Description of Studies Included in This Article}

Of the 71 studies (121 data subsets) reporting cardiovascular outcomes (Table 1): 15 were conducted in Germany; 8 in the United States; 6 in Italy; 4 each in the Netherlands and Spain; 3 in the United Kingdom; 2 each in Canada, Switzerland, Australia, Belgium, Denmark,
France, Mexico, Poland, Tunisia, and Venezuela; and 1 each in Brazil, Chile, Finland, Guadeloupe, Iceland, Israel, Kuwait, Norway, Portugal, the Philippines, and Thailand. Most studies defined obesity as having a BMI-SDS $>2$ or a BMI percentile of at least the 95th percentile. Doughty et al. were included, as although a "BMI of at least the 89th percentile" (Table 1) is stated in their methods, ${ }^{51}$ the paper reports on 12 adolescents with obesity, with BMIs above the 95th percentile completing the intervention; and BMISDS was $2.3 \pm 0.3$ at baseline - therefore an obese sample. ${ }^{19,20}$

Most included studies were of cohort design $(n=49)$, and 18 were RCTs. There was also one case-control study, ${ }^{48}$ a nonrandomized prospective study, ${ }^{90}$ and a controlled clinical trial. ${ }^{112}$ One study adopted a quasi-randomized design, whereby two cohorts were partially randomized to either an intervention group or a "usual care" group. ${ }^{51}$

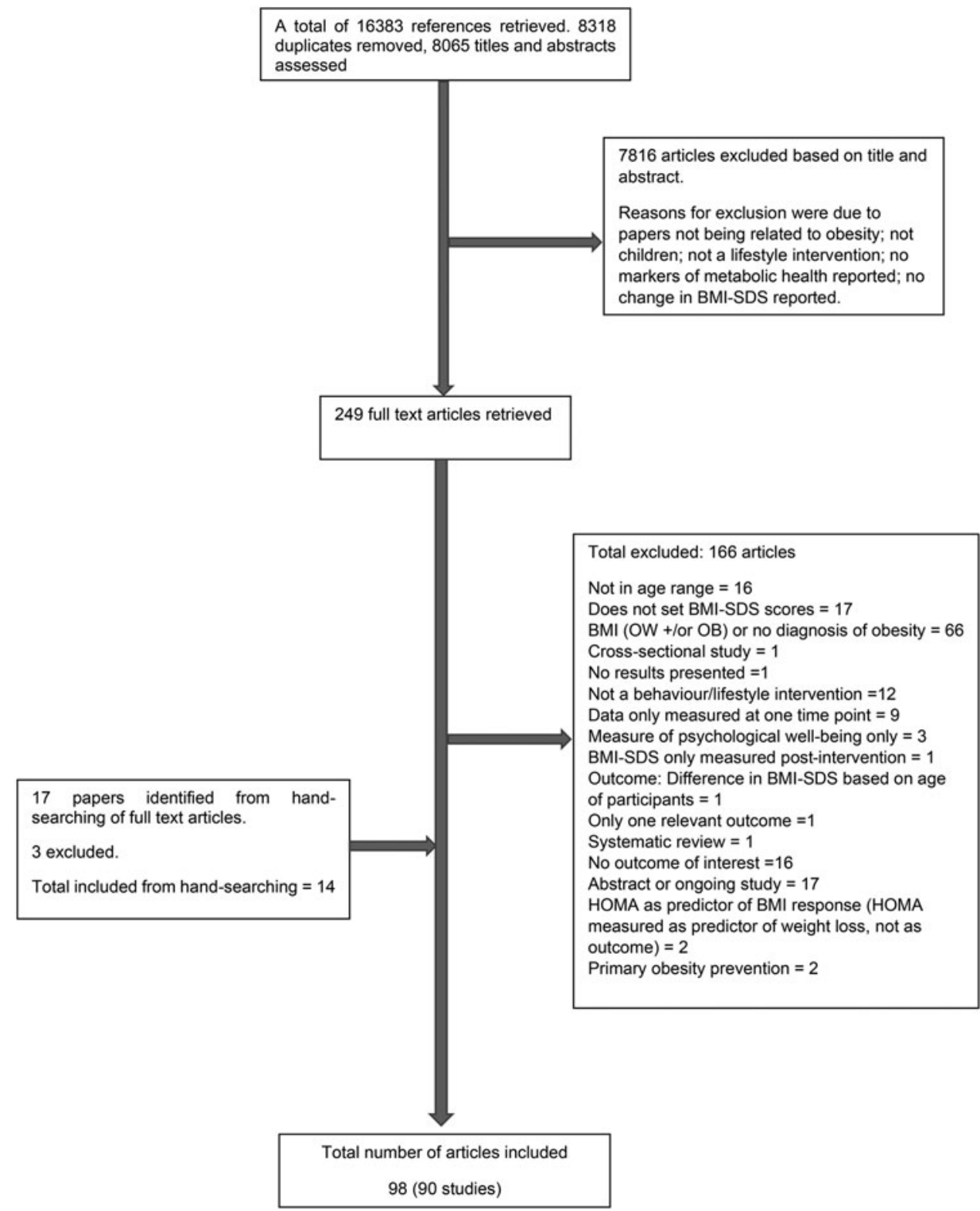

Figure 1. Flow diagram from the systematic review that identified the included studies. 


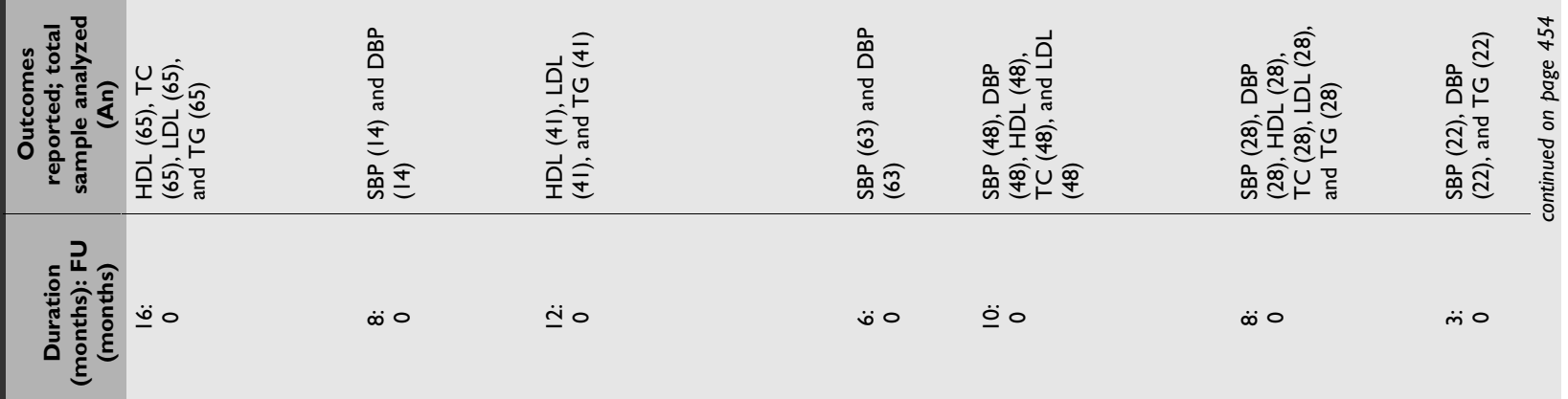

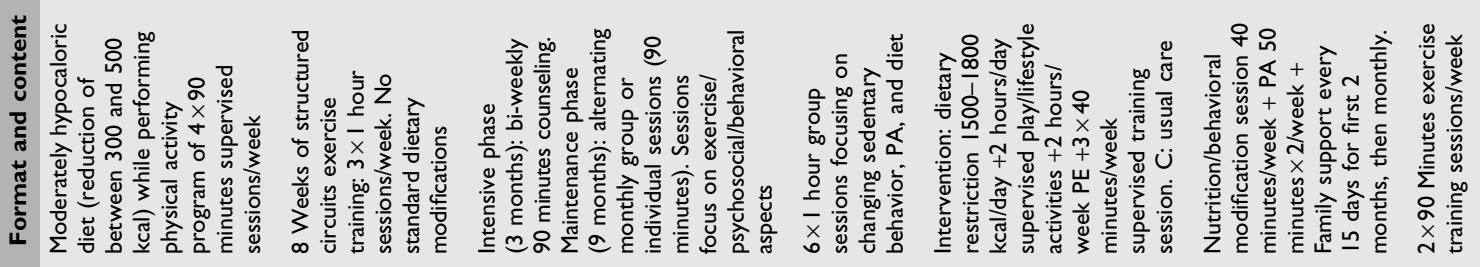

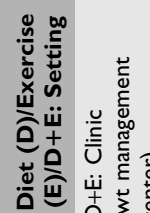

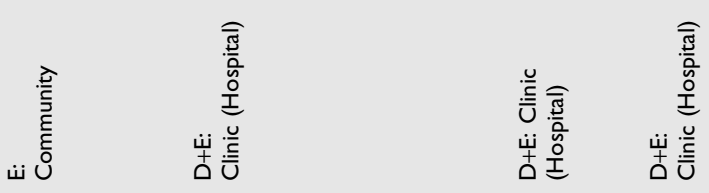

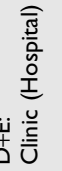

殅

ن
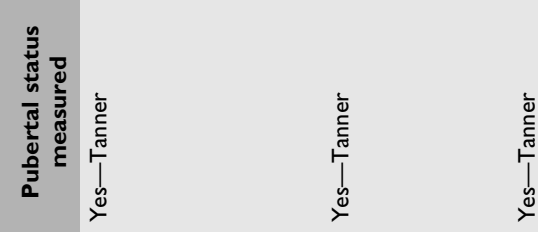

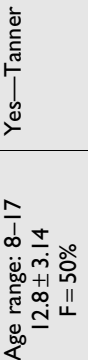

$\stackrel{o ̛ n}{z} \quad \frac{o}{z}$

兴

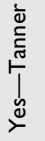

䒽希

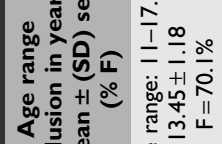

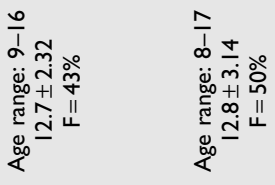

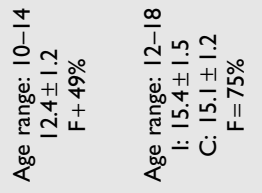

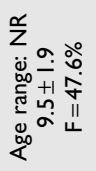

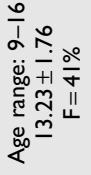

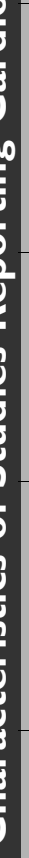

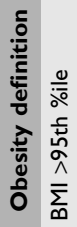

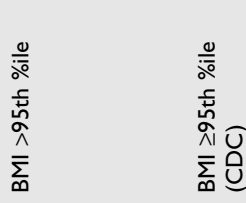

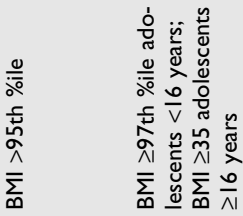

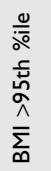
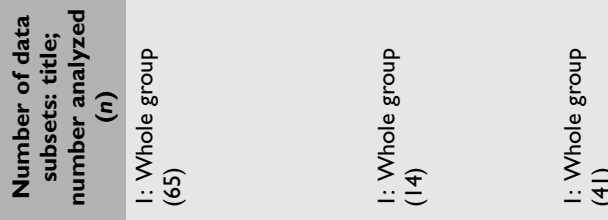

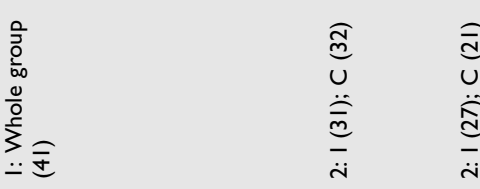
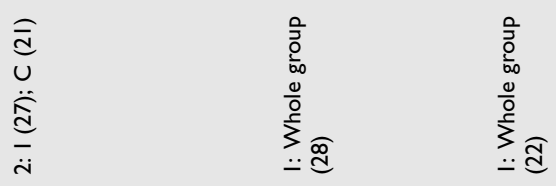

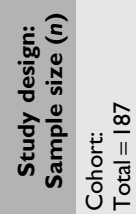

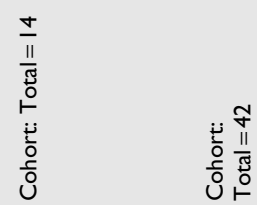

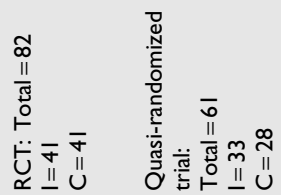
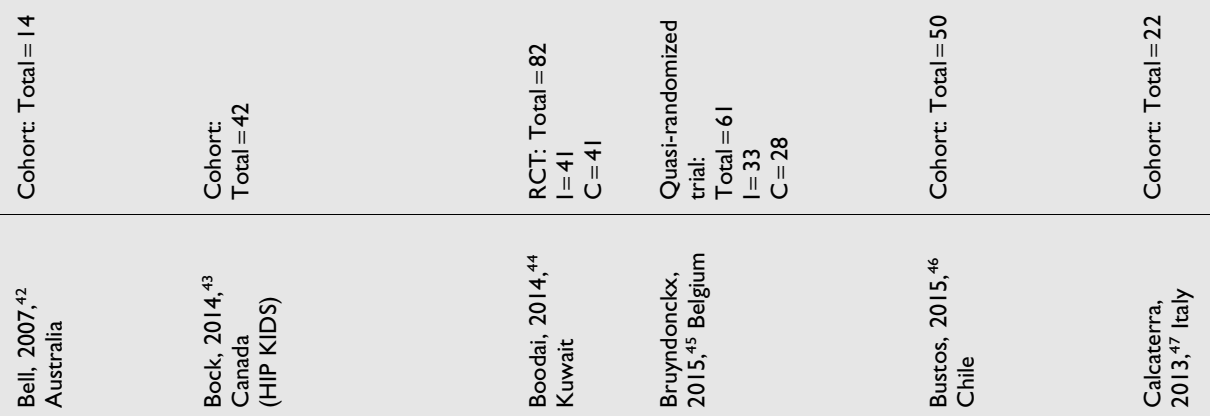

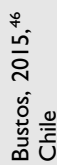


111

lis

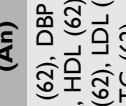

就

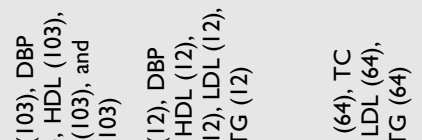

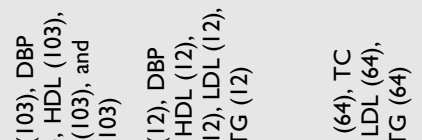

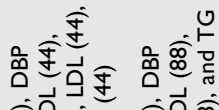

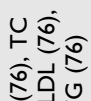

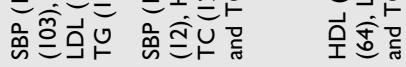

应金府

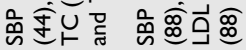

무융두

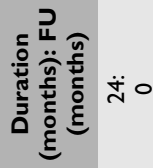

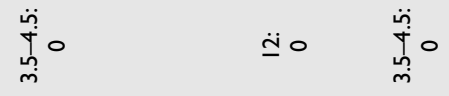

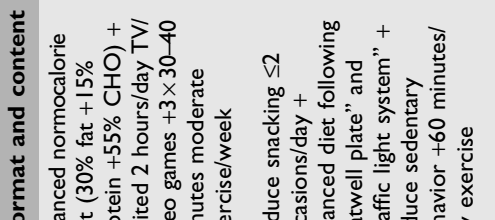

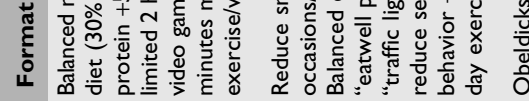

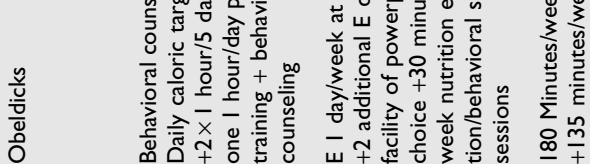

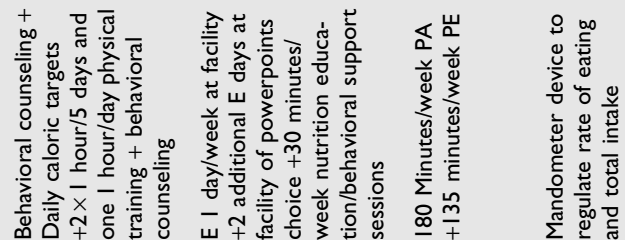

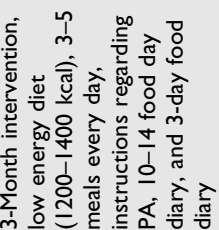

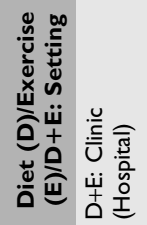

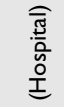

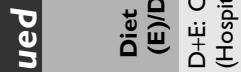

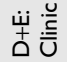

嵌を嵌

殅

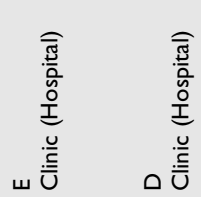

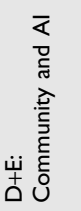

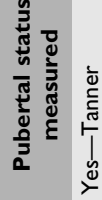

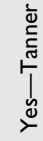

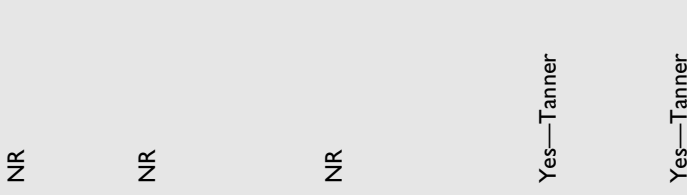

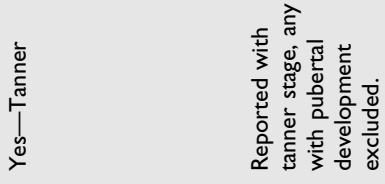

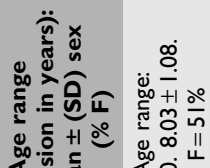

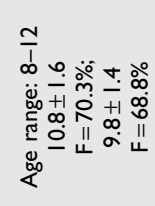

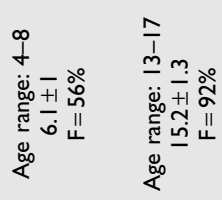

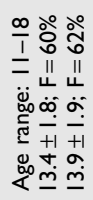

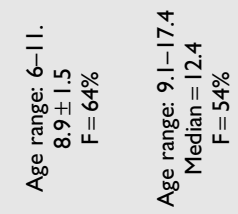

의 돈율

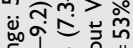

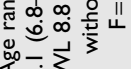

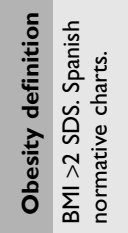

㐫 $\frac{1}{\sum_{0}}$

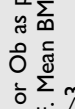

उ容㑒

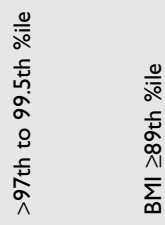

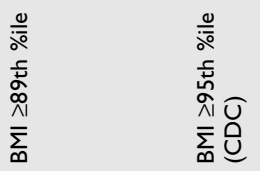

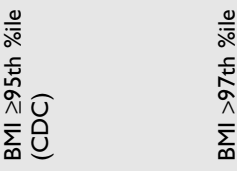

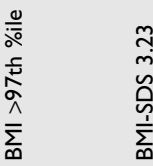

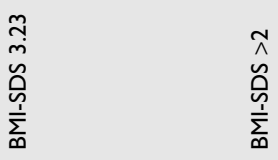

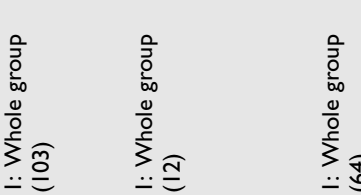

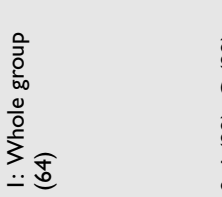

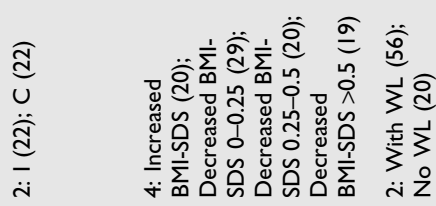

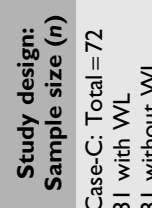

후을

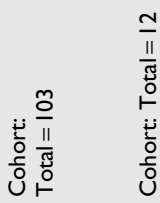

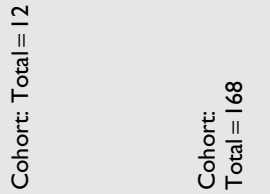

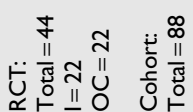

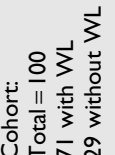

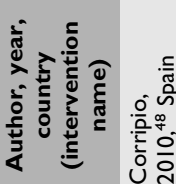

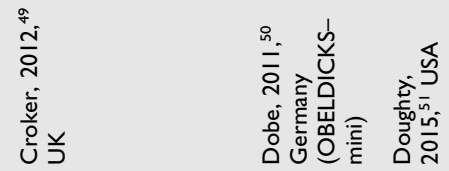

客

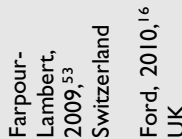

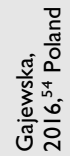

으

$=$

$\simeq$

m

$\pm$

느 


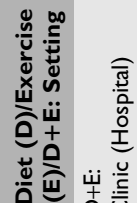

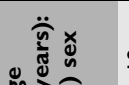

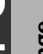

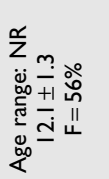

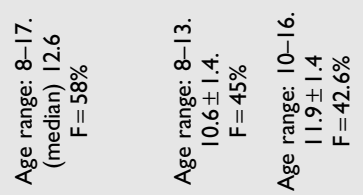

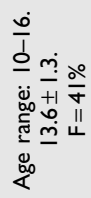

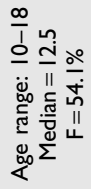

䓪

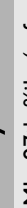

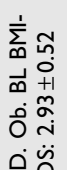

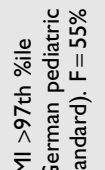

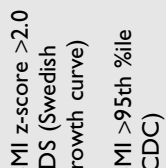

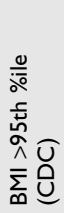

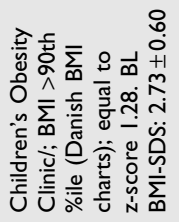

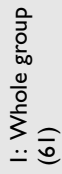

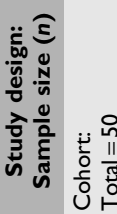

产

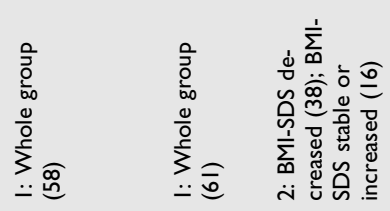

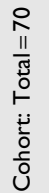

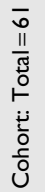

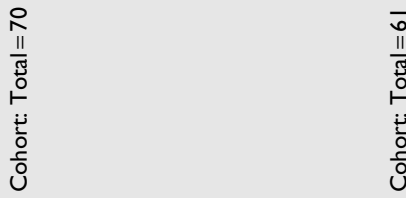

嗹

就高部

留

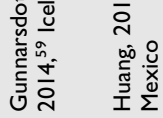

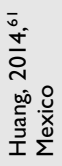

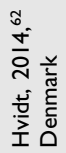

$\underline{\infty}$

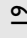

ㅇำ

$\bar{N}$

ส 


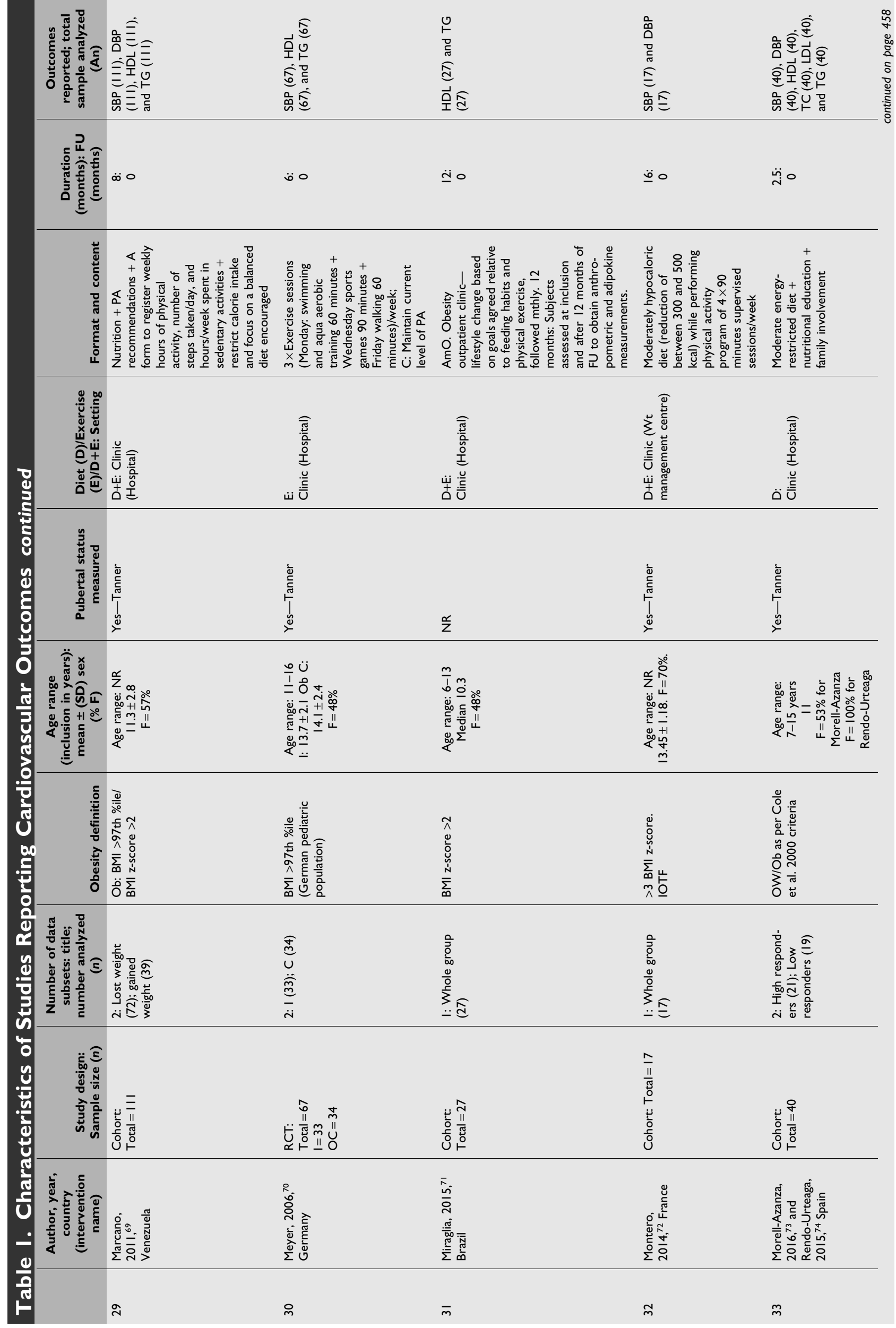




\begin{tabular}{|c|c|c|c|c|c|c|c|}
\hline 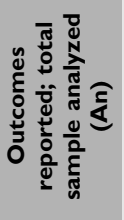 & 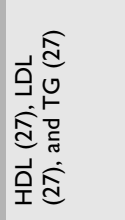 & 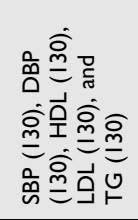 & 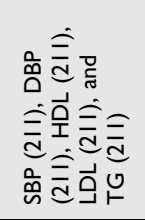 & 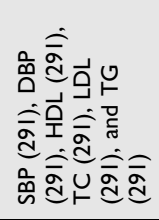 & 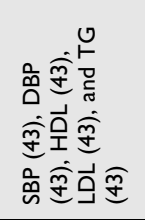 & 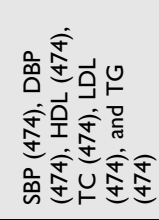 & 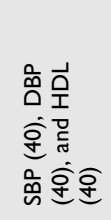 \\
\hline 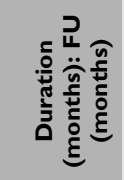 & $\ddot{\underline{O}} 0$ & घ̈o & ت̇o & ভ̇o & غ̇o & ن்o & $\dot{\underline{y}} 0$ \\
\hline 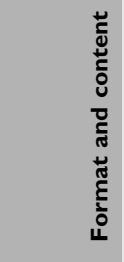 & 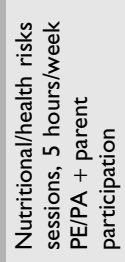 & 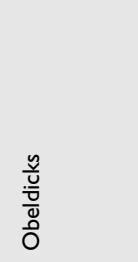 & 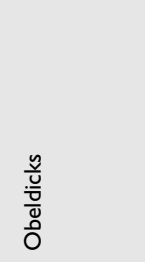 & 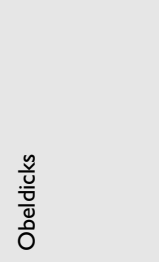 & 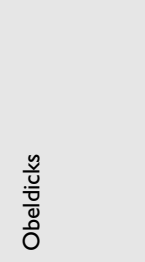 & $\begin{array}{l}\frac{\tilde{v}}{\dot{v}} \\
\frac{\tilde{v}}{\nu} \\
0\end{array}$ & 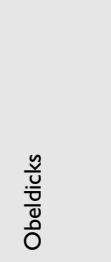 \\
\hline
\end{tabular}
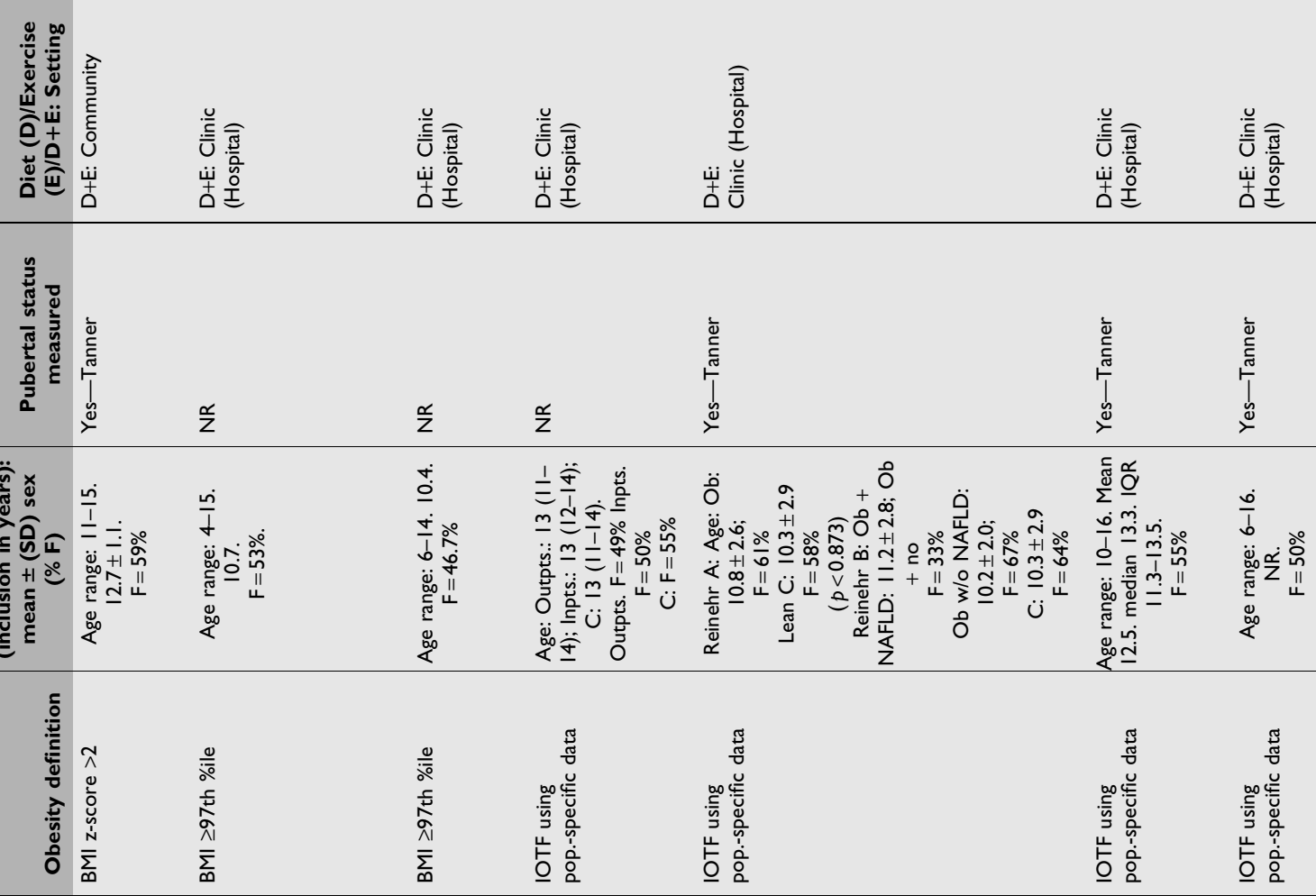

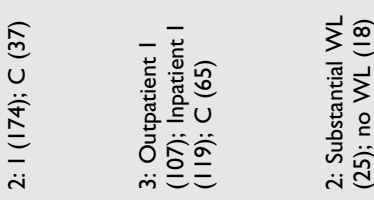
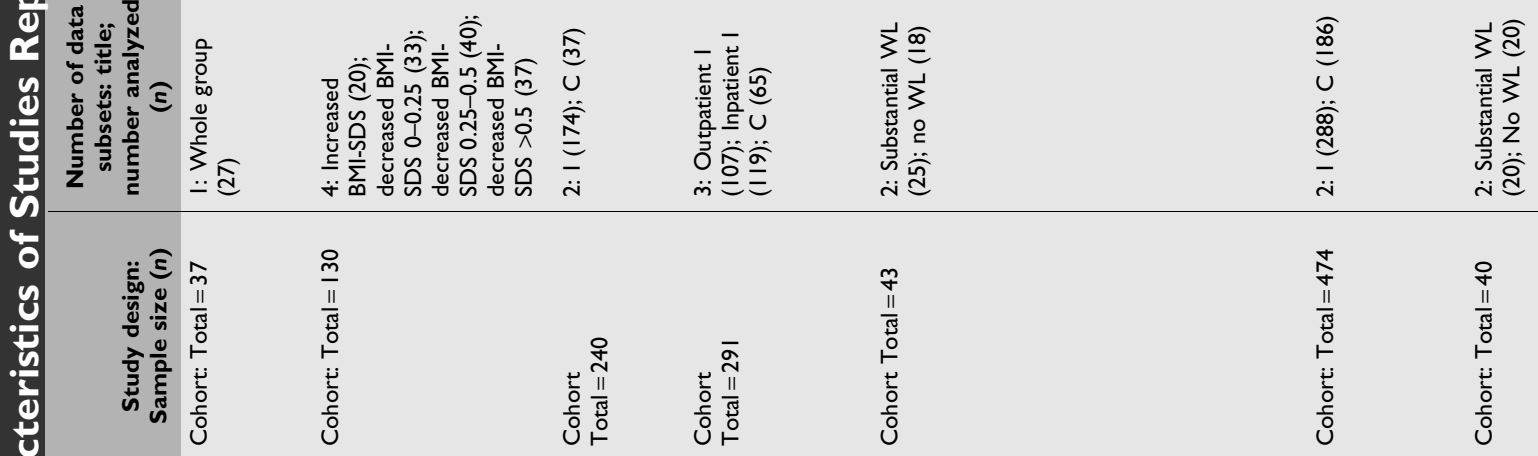

$m$
$\frac{m}{11}$
$\frac{1}{5}$
$\circ$
$\frac{5}{0}$
0
0
0
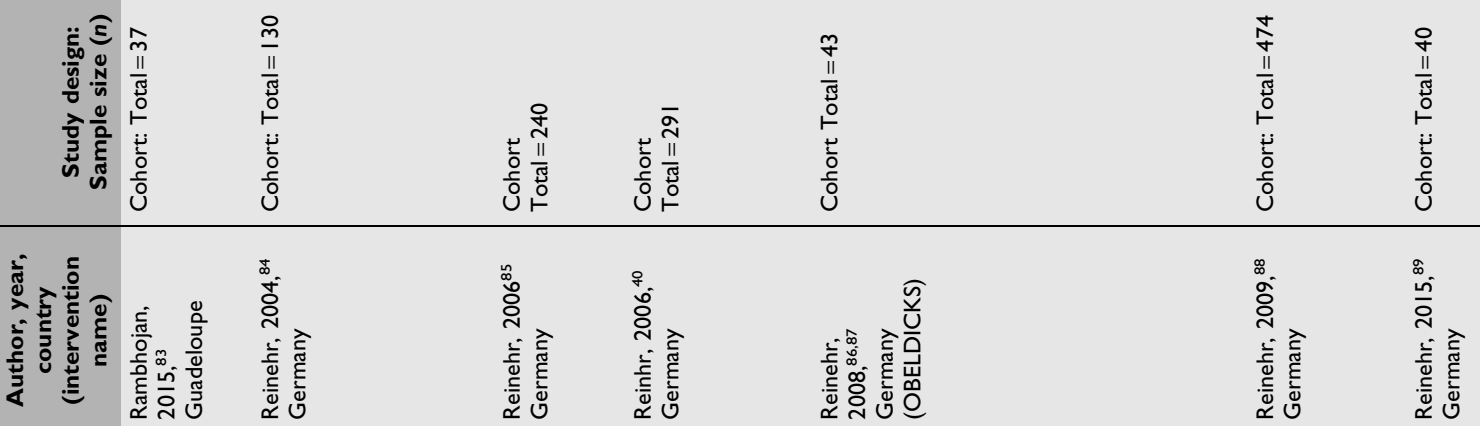


\begin{tabular}{|c|c|c|c|c|c|}
\hline 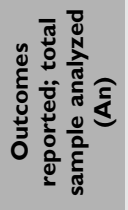 & 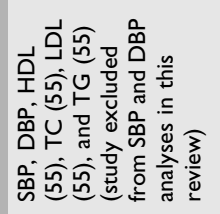 & 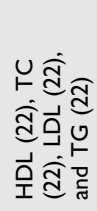 & 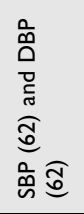 & 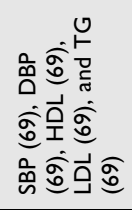 & 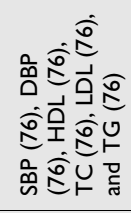 \\
\hline 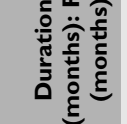 & ザ० & घ்o & ஹ்o & घ்o & ஸ்o \\
\hline
\end{tabular}

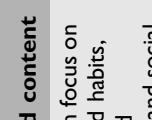

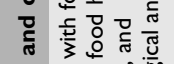

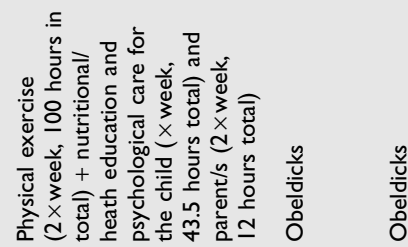

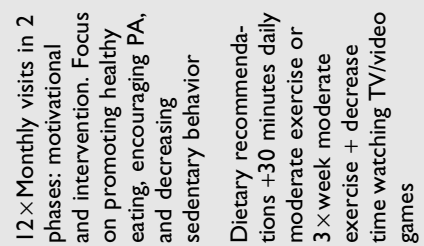

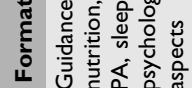

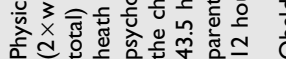

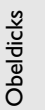
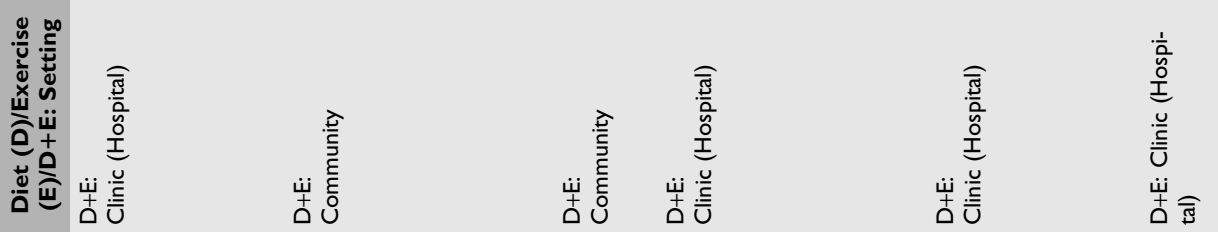

\section{㸺}

ž

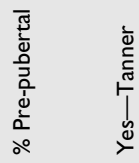

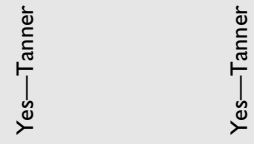

\section{䒽}

$\ddot{\circ}$

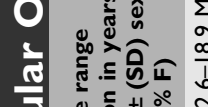

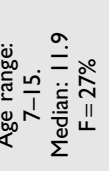

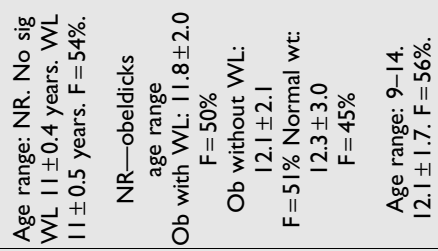

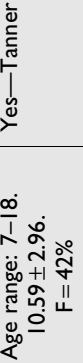

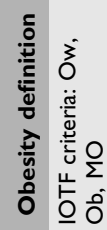

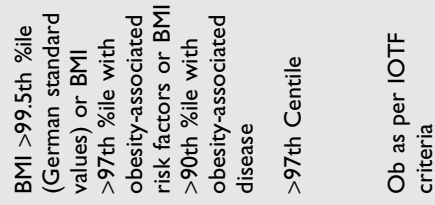

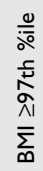

क응

去

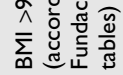

ปั)

نำ

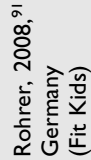

g

요

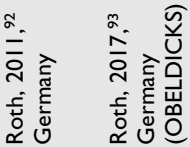

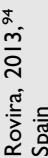

ก

n

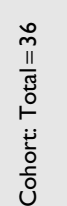

[!n 


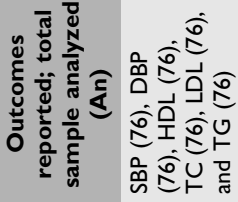

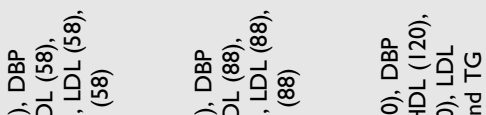

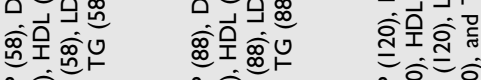

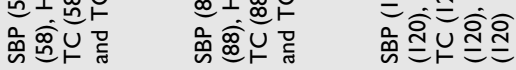
于
商

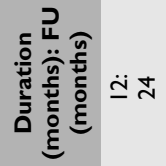
$\ddot{\circ}$
$\ddot{\circ}$
$\ddot{m} \sigma$
$\ddot{m} 0$

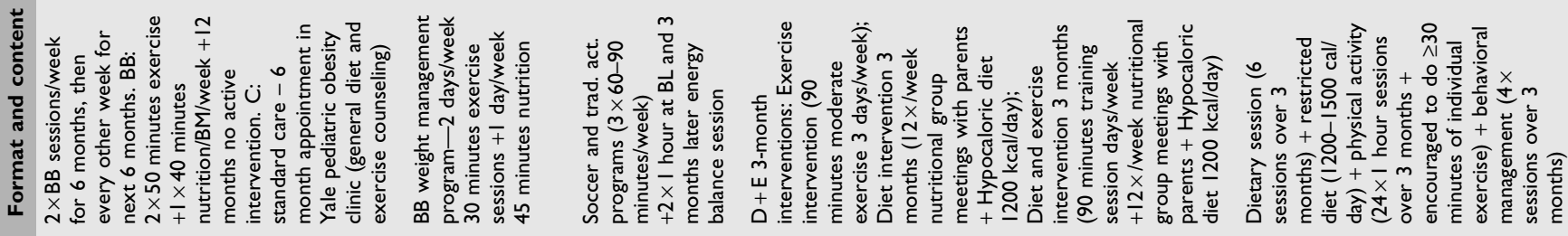

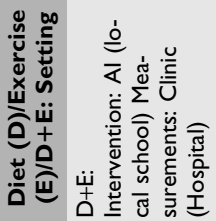
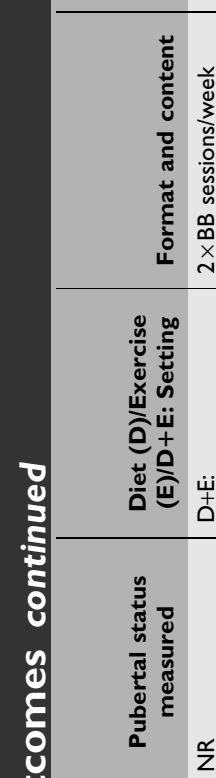

殅を

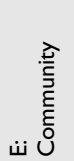

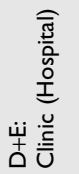

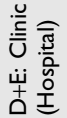

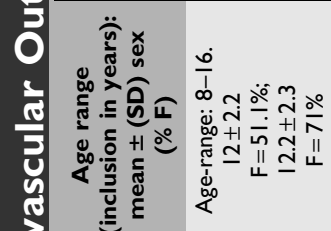



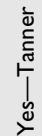

$\stackrel{\propto ⿻ 上 丨}{z}$

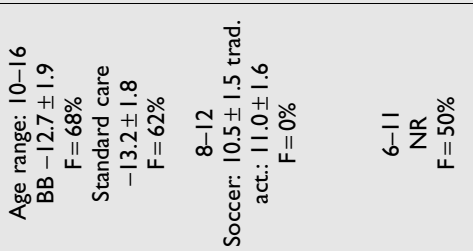
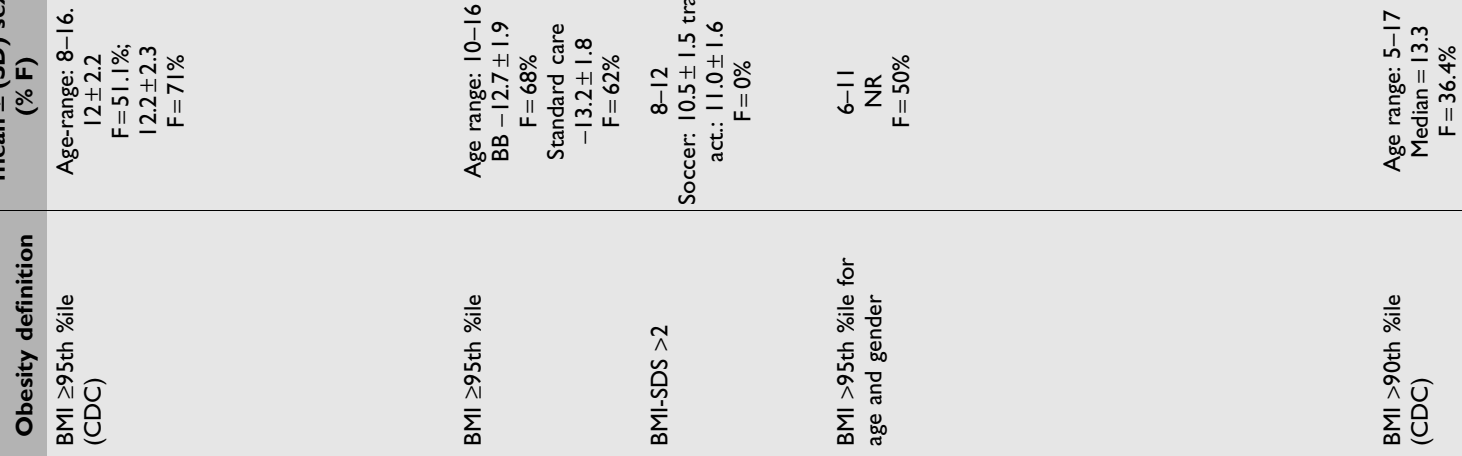

\begin{tabular}{ll}
\hline \\
0 \\
0
\end{tabular}

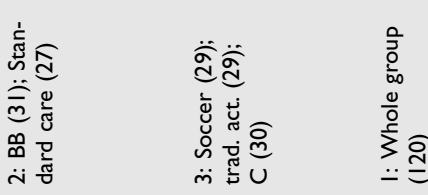

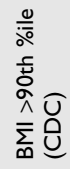

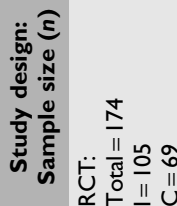

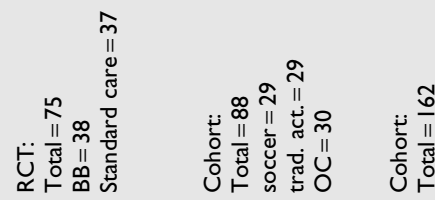

نำ

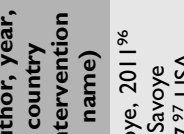

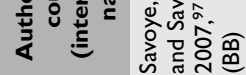

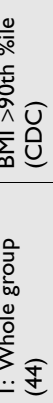

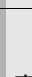

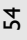

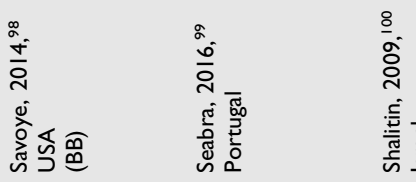

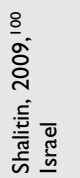

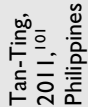

นกำ

เீ

กิ

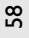



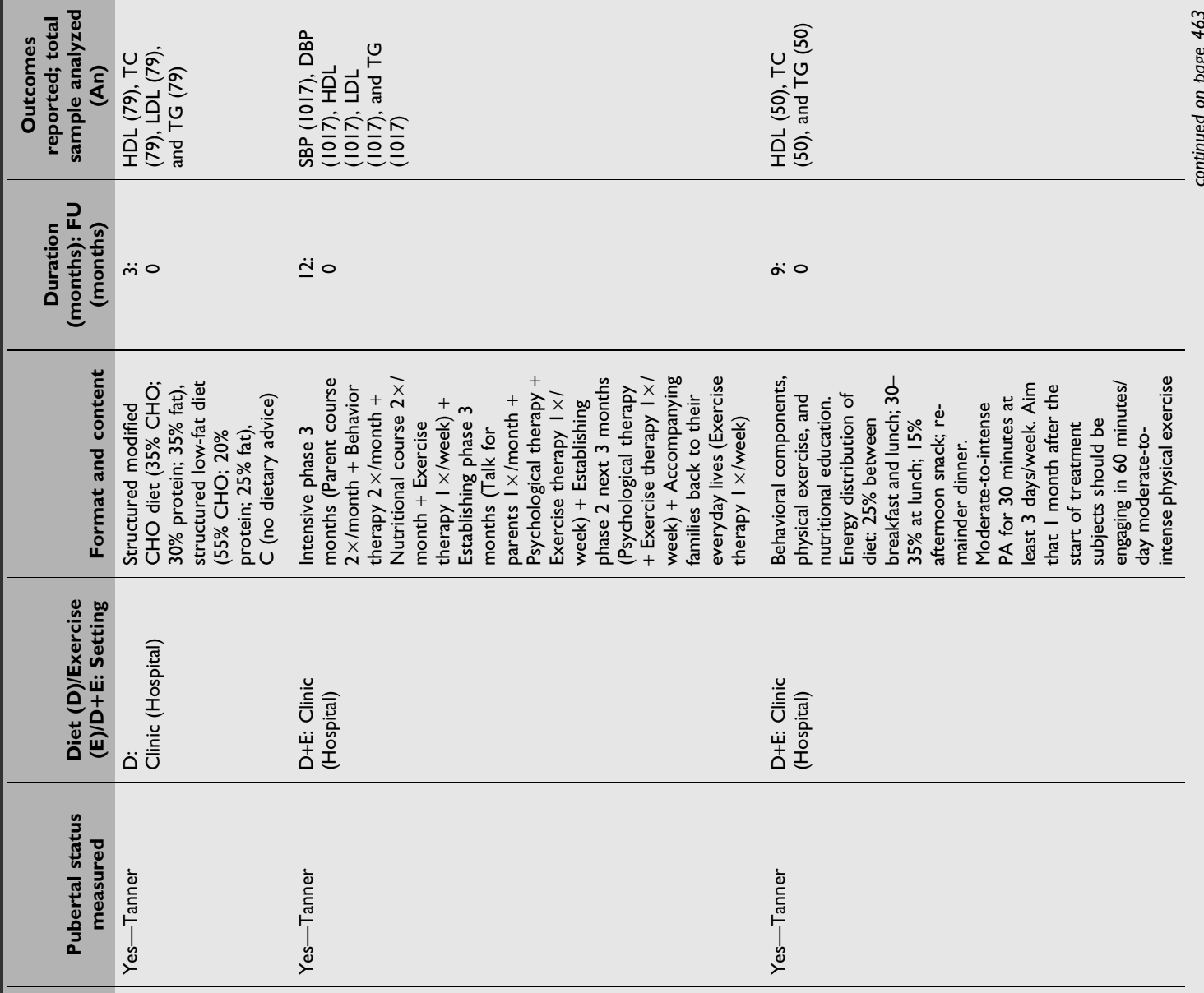

\begin{tabular}{|c|c|c|}
\hline 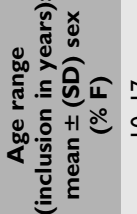 & 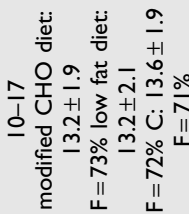 & \\
\hline
\end{tabular}

\begin{tabular}{|c|c|}
\hline 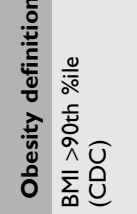 & 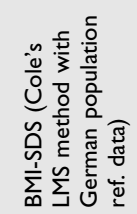 \\
\hline
\end{tabular}

\begin{tabular}{|c|c|c|}
\hline 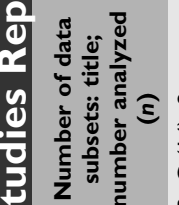 & 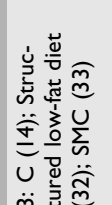 & 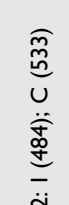 \\
\hline
\end{tabular}

\begin{tabular}{|c|c|c|}
\hline 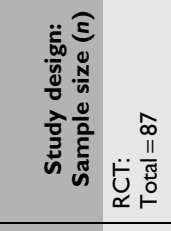 & 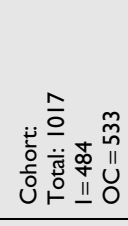 & 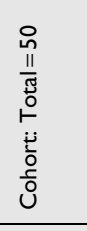 \\
\hline 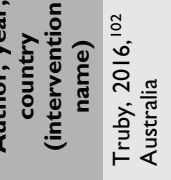 & 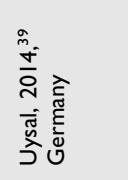 & 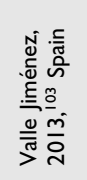 \\
\hline in & 8 & $\overline{0}$ \\
\hline
\end{tabular}




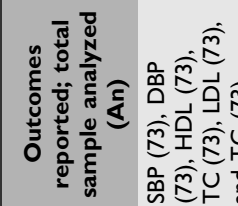

U

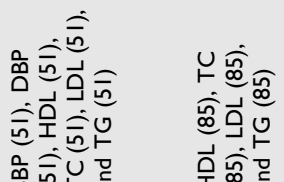

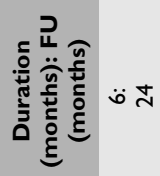

j̆o

ĩo

$\ddot{\leftrightarrow}$

ปั้ ำ ำ

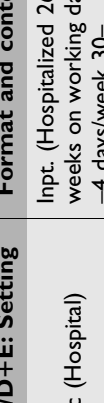

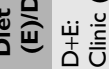

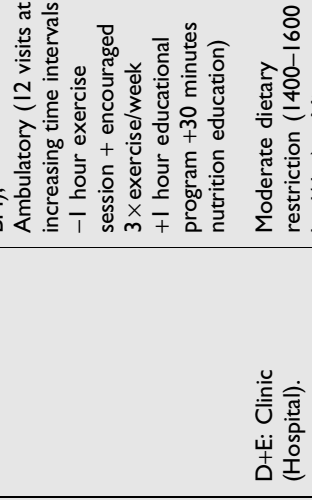

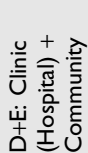

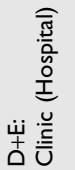

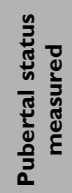

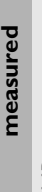

+ 岛

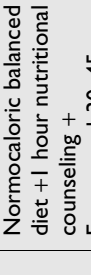

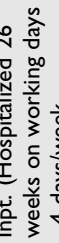

8.

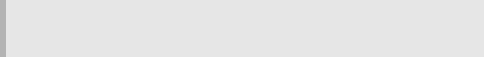

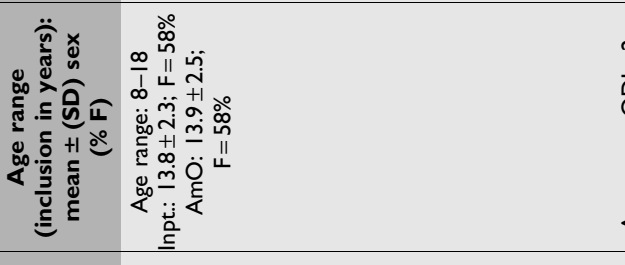

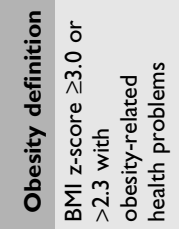

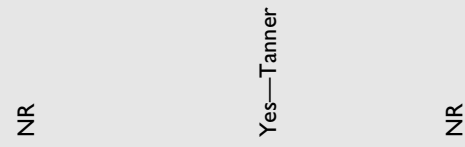
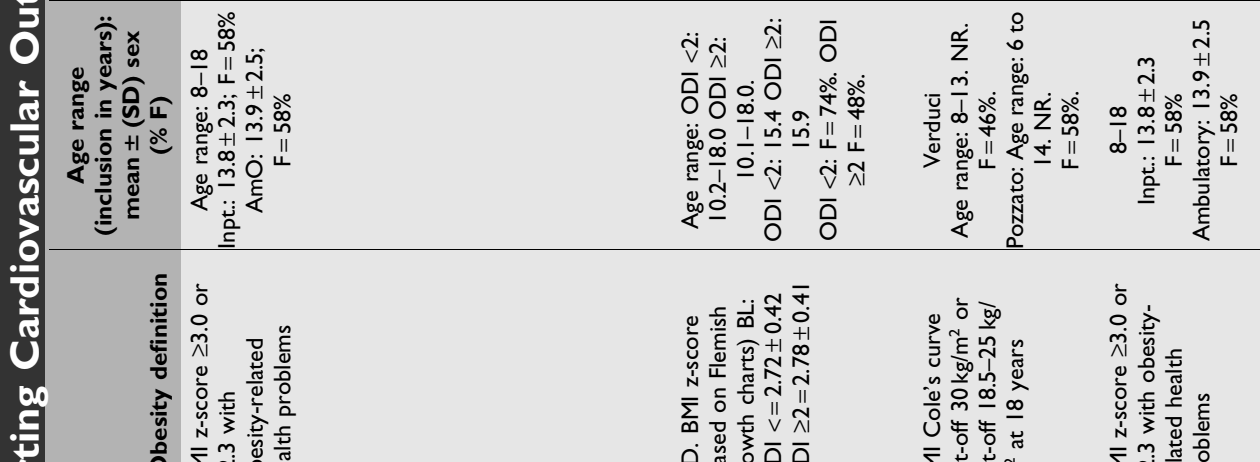

일

的 $\lambda$ 용

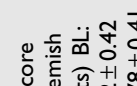

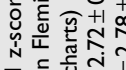

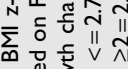

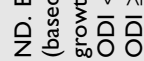

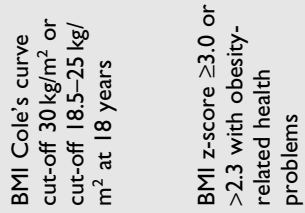

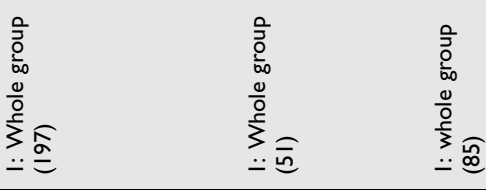

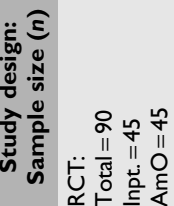

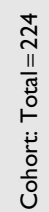

ำ

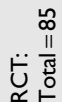

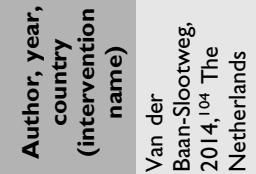

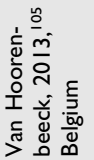

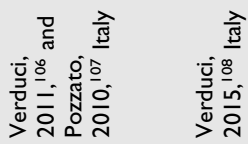

3

t5

ถุ 


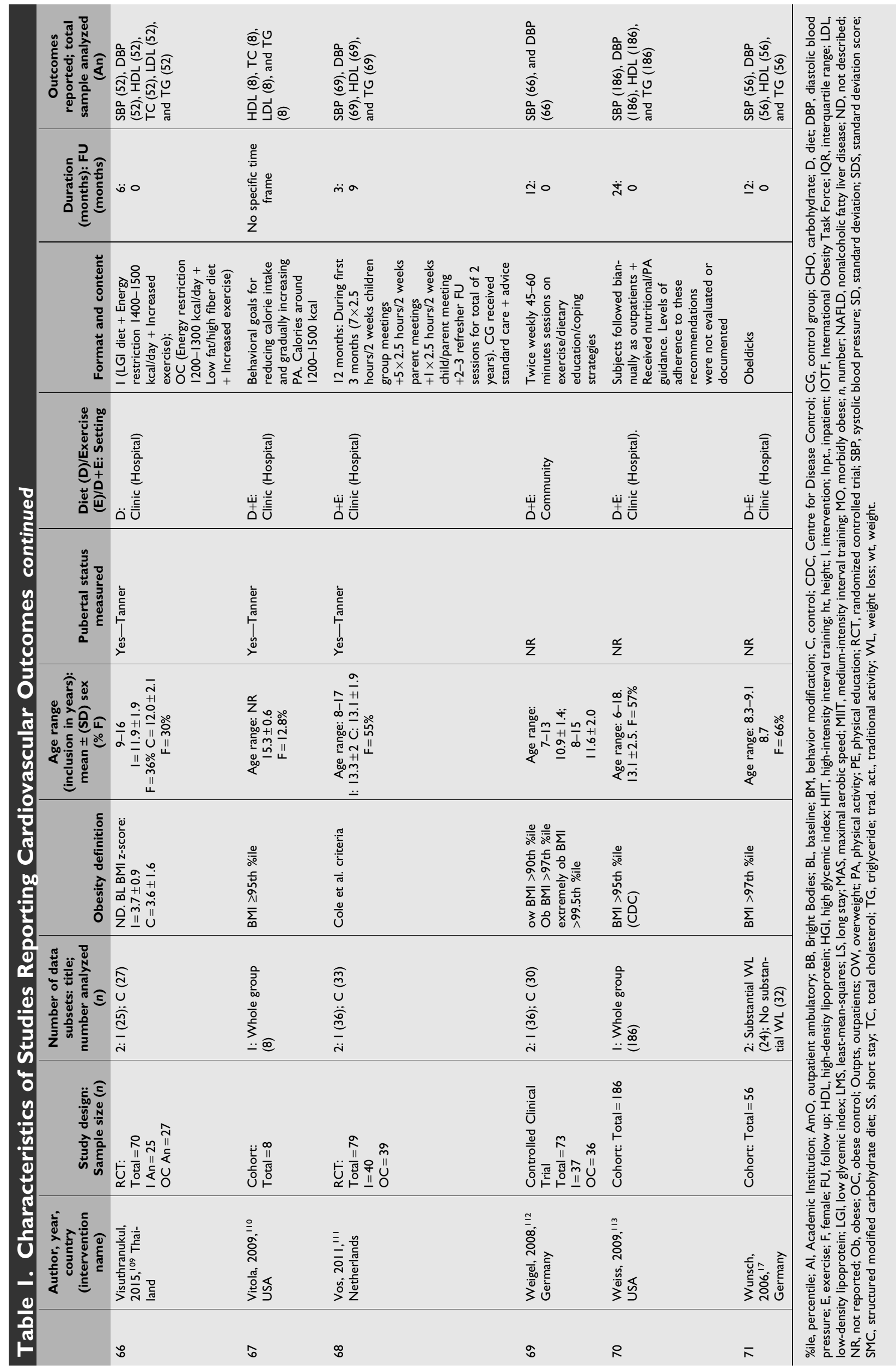


Most interventions were conducted in a hospital clinic setting $(n=50)$. Ten studies were undertaken in a community setting and eight in academic institutions. Three studies conducted their intervention between academic and clinical settings, ${ }^{96}$ clinical and community settings, ${ }^{106}$ or community and academic settings. ${ }^{54}$

Fifty-nine studies conducted interventions that comprised both diet and exercise components. The remaining studies $(n=12)$ utilized either exercise or diet interventions only. The duration of the interventions ranged from 2 to 24 months, with one study having no specific intervention period. ${ }^{116}$ Most studies $(n=65,90 \%)$ did not report any follow-up after the lifestyle treatment post-intervention assessment. Follow-up ranged from 6 to 24 months, in studies where it was conducted and reported.

The sample sizes of the included studies ranged from 8 to 1017 participants. Ages of participants ranged from 4 to 19 years. Studies predominantly had a mix of men and women ( $n=67,94 \%$ ), with only four studies specifically analyzing either girls ${ }^{81,82}$ or boys. ${ }^{74,99}$ Thirty-seven studies $(53 \%)$ measured the pubertal development stage of participants, according to the Marshall and Tanner staging, with pubertal status categorized into three groups: prepubertal, pubertal, and late/post-pubertal. ${ }^{114}$ One study reported that pubertal development was measured, although the method used was not defined, ${ }^{79}$ and one study reported the percentage pre-pubertal, without defining how they measured puberty. ${ }^{92}$ Some studies did not report any measures of pubertal development $(n=32,45 \%)$.

\section{Quantitative Analysis}

Systolic blood pressure. Table 1 shows the number of subsets included from each study, and the number analyzed from each subset. Before SBP analysis, two studies $(n=4$ subsets) were excluded as they reported SBP-SDS and were not directly comparable. ${ }^{49,90}$ As a result, 99 subsets were identified from the remaining 54 studies that documented both a mean change in BMI-SDS and an associated mean change in SBP (or gave pre- and post- "intervention" values from which these could be calculated), together with the number of cases analyzed. "Intervention" here could have been the "control" arm in some cases. Two studies ( $n=5$ subsets) only had medians and IQRs reported for the baseline and post-intervention results; the mean and SDs were estimated from them. ${ }^{39,40}$

Thirty data subsets had the mean change in SBP reported, together with a measure of variability (standard deviation [SD]) of the actual changes; an initial analysis of just these 30 subsets is shown in Appendix A2 and Appendix Figure A1(A). The remaining 69 subsets reported means and SDs (or SEs, or 95\% CIs) at baseline and "end," and the mean and SD of the changes were calculated from these. The latter required an estimate of the correlation between baseline and end SBP; we used the median correlation coefficient estimated from 15 datasets reporting SDs of both changes and baseline/post- intervention [0.66 (IQR 0.54-0.71)]. The final analysis for all 99 subsets and the fitted meta-regression line is shown in Figure 2, together with pointwise, 95\% PIs for the change in SBP across the study datasets. The equation of the fitted line was: Mean change in $\mathrm{SBP}=-6.153 \times$ mean change in BMI-SDS +1.408 . The mean changes in SBP and BMI-SDS were significantly related as indicated by the regression slope $(p<0.001$, with $95 \% \mathrm{CI}:-8.787$ to $-3.520)$. However, from the $95 \%$ PI, a mean change of at least 1 in mean BMI-SDS would be required to ensure a reduction in SBP in any future study.

Appendix Figure A1(B) shows the half normal plot of the standardized predicted random effects, most of which are within \pm 2 . The proportion of the between-study variance that was explained by the mean change in BMI-SDS was only $22 \%$. There was significant variation due to heterogeneity $\left(I^{2}=89 \%\right)$. Further exploratory analyses, using the percentage of women and the length of the study (baseline to end-of-intervention) as additional predictors, failed to show any significant effect on the mean change in $\operatorname{SBP}(p=0.24$ and $p=0.27$, respectively).

HDL cholesterol. Before analysis, Kolsgaard et al. $(n=4)$ was excluded as the mean change in BMI-SDS was reported for the whole sample size (0.13) and not the specific subsets in the study ${ }^{66}$ Therefore, 104 subsets from the remaining 59 studies reported both a mean change in BMI-SDS and an associated mean change in HDL or gave pre- and post- "intervention" values from which these could be calculated as well as the number of cases analyzed. Thirty-eight subsets reported HDL in $\mathrm{mmol} / \mathrm{L}$ - the international system of units. The remaining $(n=66)$ required their reported values to be multiplied by 0.02586 to convert them from $\mathrm{mg} / \mathrm{dL}$ to $\mathrm{mmol} / \mathrm{L}{ }^{115}$ Six studies $(n=11)$ reported medians and IQRs for the baseline and post-intervention results. ${ }^{39,40,54,75,76,105}$

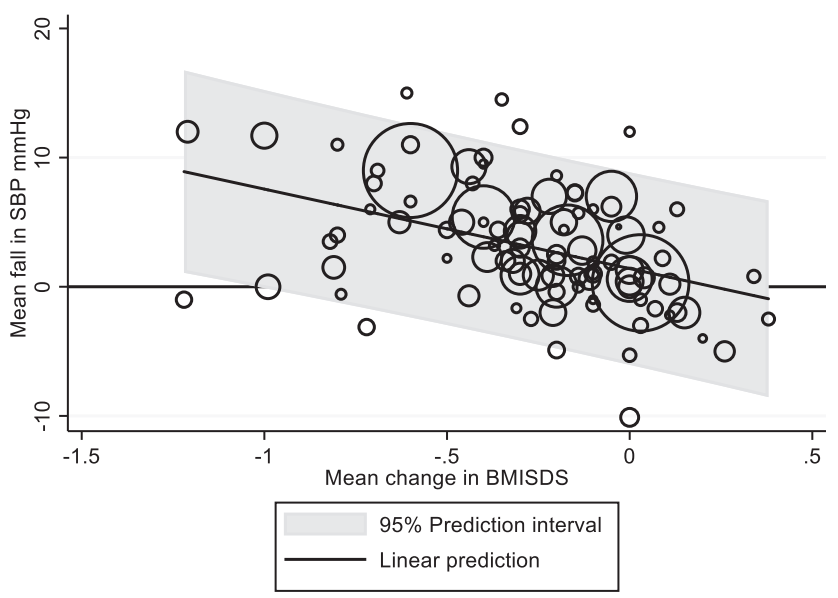

Figure 2. Meta-regression showing the relationship between mean change in BMI-SDS and SBP $(n=99)$. SBP, systolic blood pressure; SDS, standard-deviation score. 
Twenty-five subsets reported mean and SDs of the changes in HDL and analysis based on those given in Appendix Fig. A2(A). The median of the correlation coefficients estimated from 16 datasets reporting SDs of mean change, baseline and post-intervention HDL values was 0.60 (IQR 0.31-0.79), which was used to estimate the SDs of the changes in HDL for the remaining 78 subsets, leaving 104 subsets for analysis (Appendix Fig. A2(B)). In Figure 3, however, the control subset in Visuthranukul et al. has been omitted $(n=103)$ because it was found to be a strong outlier in the analysis, with a mean reduction of $1.1 \mathrm{mmol} / \mathrm{L}^{109}$ in HDL (Appendix Fig. A2(B)). The equation of the fitted line was: mean change (increase) in $\mathrm{HDL}=-0.065 \times$ mean change in BMI-SDS +0.009 . (adjusted $\left.R^{2}=9 \% ; I^{2}=86 \%\right)$. Although the slope was statistically significant ( $p=0.009 ; 95 \% \mathrm{CI}-0.112$ to -0.017 ), the change in BMI-SDS needed to ensure an increase in mean HDL was beyond the range of the data (in excess of 1.22). The standardized predicted random effects were approximately normal (Appendix Fig. A2(C)). Including the control subset in Visuthranukul et al. ${ }^{109}(n=104)$ did not impact this result, in that it was still impossible to find a change of BMI-SDS, among the range of changes seen, that would ensure an improvement in mean HDL (Appendix Fig. A2(B)).

Neither the percentage of women nor the length of the study (baseline to end-of-intervention), added as extra predictors, significantly affected the mean change in HDL $(p=0.86 ; p=0.057)$.

LDL cholesterol. Before the LDL analysis, Kolsgaard et al. ${ }^{66}$ was excluded for similar reasons as the HDL analysis. Eighty-two data subsets from the remaining 46 studies reported both a mean change in BMI-SDS and an associated mean change in LDL or gave pre- and postintervention values from which these could be calculated,

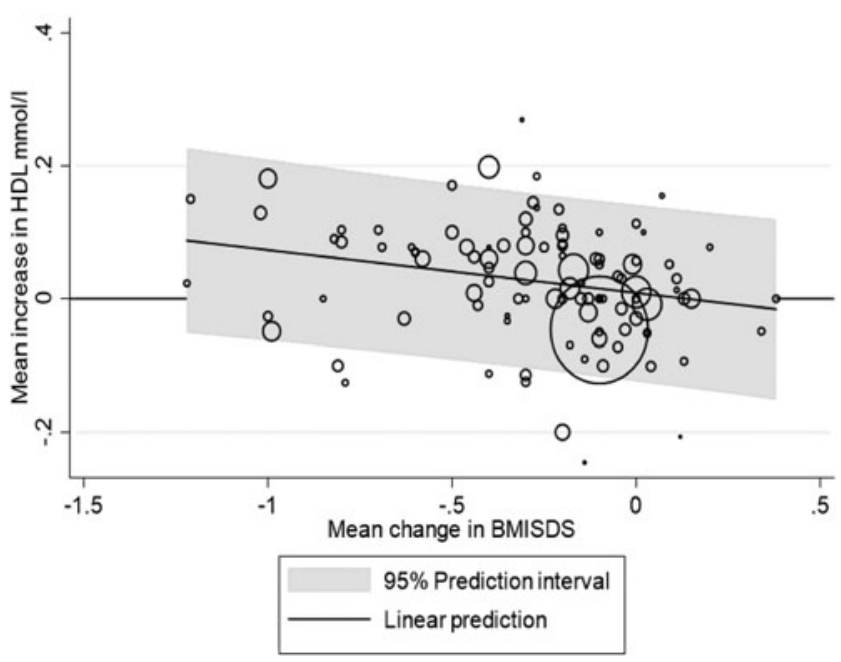

Figure 3. Meta-regression showing the relationship between mean change in BMI-SDS and HDL $(n=103$ excluding the outlier). HDL, high-density lipoprotein. together with the number of cases analyzed. Thirty-two subsets reported LDL in $\mathrm{mmol} / \mathrm{L}$. The remaining $(n=50)$ required their reported values to be multiplied by 0.02586 to convert them from $\mathrm{mg} / \mathrm{dL}$ to $\mathrm{mmol} / \mathrm{L}$. ${ }^{115}$ Seven subsets reported medians and IQRs for the baseline and postintervention results. ${ }^{39,40,54}$

Twenty-four subsets reported a mean change in LDL together with SDs (Appendix Fig. A3(A)). The median and IQR of the correlation coefficients estimated from the 15 datasets where the SDs of mean change, baseline and postintervention LDL values were available was 0.80 (IQR 0.66-0.83), which was used to estimate the SDs of the changes in LDL for the remaining 58 subsets and the overall meta-regression line is shown in Figure 4. The equation of the line was: Mean change in $\mathrm{LDL}=-0.225 \times$ Mean change in Mean BMI-SDS +0.101. (adjusted $\left.R^{2}=11 \% ; I^{2}=94 \%\right)$. The slope was statistically significant ( $p=0.002$; $95 \% \mathrm{CI}-0.362$ to -0.088$)$; however, looking at the $95 \%$ PI, the mean BMI-SDS would have to be reduced by at least 1.2 to ensure a reduction in mean LDL. Murer et al. $^{76}$ was a strong outlier, although omission of this subset $(n=81)$ did not change the overall findings (Appendix Fig. A3(B)).

The standardized predicted random effects were approximately normal (Appendix Fig. A3(C)). Neither the percentage of women nor the study duration significantly affected the mean change in LDL when added to the regression model ( $p=0.83$ and $p=0.16$, respectively, $n=81)$.

Triglycerides. Before the TG analysis, Kolsgaard et al. ${ }^{66}$ was excluded for similar reasons as the previous analyses. The weight-loss subset in Corripio et al. ${ }^{48}(n=1)$ was excluded, as the reported baseline IQR for TG was incorrect. Savoye et al. ${ }^{96}(n=2)$ was excluded, as the changes from baseline TG were reported as CIs of geometric means (GMs) from which it was impossible to determine SDs for the actual changes in TG. After these

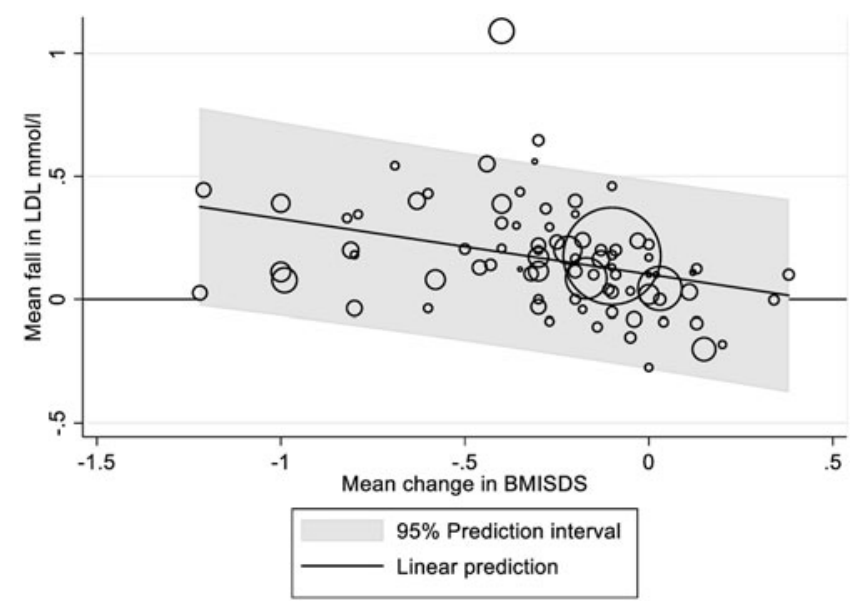

Figure 4. Meta-regression showing the relationship between mean change in BMI-SDS and LDL $(n=82)$. LDL, low-density lipoprotein. 
exclusions, 98 data subsets from 54 studies reported both a mean change in BMI-SDS and an associated mean change in TG or gave pre- and post-intervention values from which these could be calculated, as well as the number of cases analyzed. Thirty-six subsets reported TG in $\mathrm{mmol} / \mathrm{L}$; the remaining $(n=62)$ required their reported values to be multiplied by 0.01129 to convert them from $\mathrm{mg} / \mathrm{dL}$ to $\mathrm{mmol} / \mathrm{L} .{ }^{115}$ Nine subsets reported medians and IQRs, ${ }^{39,40,48,54,71}$ and two subsets reported medians and ranges ${ }^{76,105}$ for both the baseline and post-intervention results. For Ford et al. $(n=4)$, who reported TG values as GMs and ranges, GMs were used to approximate the medians and the mean/SD was estimated from the medians/ranges. ${ }^{16}$

Twenty subsets reported a mean change in TG together with SDs (Appendix Fig. A4(A)). The median and IQR of the correlation coefficients estimated from the 13 datasets where the SDs of mean change, baseline and postintervention TG values were available was 0.69 (IQR 0.53-0.78), which was used to estimate the SDs of the changes in TG for the remaining 78 subsets The metaregression for all 98 subsets is shown in Figure 5. The equation of the fitted line was: mean change in $\mathrm{TG}=$ $-0.239 \times$ mean change in mean BMI-SDS +0.033 . The slope was statistically significant $(p<0.001 ; 95 \%$ CI -0.325 to -0.153 ). (adjusted $R^{2}=29 \% ; I^{2}=86 \%$ ).

The $95 \%$ PI suggests that a mean BMI-SDS reduction of 0.7 would be needed to ensure a reduction in mean TG. The standardized predicted random effects were approximately normal (Appendix Fig. A4(B)). As added predictors, the percentage of women and the length of the study did not significantly affect the mean change in TG $(p=0.21$ and $p=0.14$, respectively).

\section{Discussion}

\section{Summary of Main Results}

The objective of this article was to establish the minimum change in BMI-SDS required to improve BP and

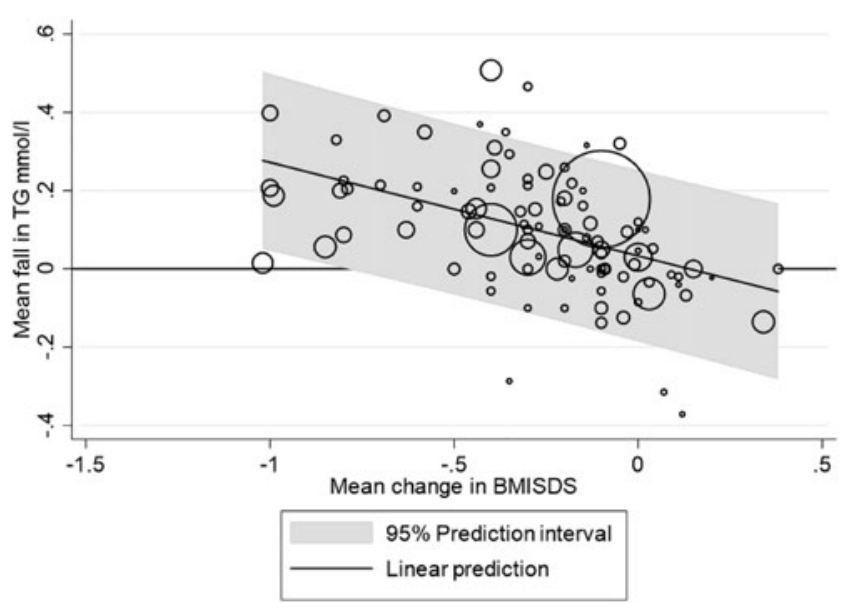

Figure 5. Meta-regression showing the relationship between mean change in BMI-SDS and TG $(n=98)$. TG, triglyceride. lipid profile in children and adolescents with obesity in a clinical trial intervention. Seventy-one of the 90 included studies reported on BP and lipid profile measurements, with 69 of the 71 studies ultimately analyzed; and SBP, HDL, LDL, and TG were included in the meta-regression. Change in BMI-SDS was significantly associated with changes in all four measurements; minimum mean reductions in BMI-SDS of $1,1.2$, and 0.7 would be needed to ensure improvements in SBP, LDL, and TG parameters, respectively, in any future clinical weight management trial.

An equivalent value for improvement in HDL could not be determined. Although there was a positive relationship between an increase in mean HDL and a reduction in BMISDS, the lower limit of the $95 \%$ PI for the increase in mean HDL was never higher than 0 within the range of BMISDS reduction seen here. A mean reduction of at least 1.22 would be needed to ensure this.

\section{Strengths and Limitations}

Currently, this is the first article to attempt to bring together all studies that have reported both a change in BMISDS and changes in BP and lipid profile measurements in the obese pediatric population. All 71 included studies were of moderate to high quality (Table 2 and Appendix A1) according to the HTA assessment tool. ${ }^{36}$

This article is the third of three papers reporting on the findings from studies identified in a large systematic review. The last study analyzed was published in September $2017,{ }^{93}$ and therefore studies published in the past 2.5 years have not been included. The first two papers in this review series were published in August and December 2019, respectively. ${ }^{31,32}$

As expected, there was a high degree of methodological and statistical heterogeneity among the included studies. This was addressed by using random-effects metaregression. Potential sources of heterogeneity include variations in sample populations with regards to number, gender, and ethnicity; pubertal status; intensity and duration of treatment and follow-up; variety of diet and exercise intervention; whether family members were involved in interventions; and the wide range of ages used in studies. Many studies did not report on all these variables, making it difficult to analyze their effect on meta-regression; however, gender and study duration were not significant confounders in the analyses shown ( $p>0.05$ for both).

Most of the studies included in this article were from the United States or Europe $(n=59)$. Unfortunately, there was a paucity of data from developing countries, and it is not possible to accurately extrapolate our results to the obese pediatric and adolescent demographics in lower-income countries. Another important factor to consider is the proportion of participants lost to follow-up. Attrition would likely have affected both change in BMI-SDS and changes in cardiovascular risk factors but would have unlikely affected the relationships between both variables. 
The systematic methods employed to identify the included studies were stringent, with the inclusion of published literature in all languages to avoid publication bias, but it is possible that some relevant studies might have been missed. In addition, there was variation in the reporting of results where there were multiple publications of the same study; in these cases, results from the most comprehensive paper were used.

At extremes of body weight, the use of BMI-SDS is another important limitation to address. Vanderwall et al. describe BMI-SDS as a poor predictor of adiposity in children and adolescents with extreme obesity, particularly those younger than 9 years of age. ${ }^{116}$ Alternative measures of pediatric adiposity, such as the percentage above the International Obesity Task Force 25 (IOTF-25) cut-offan established BMI cut-off-have been described to be more accurate than BMI-SDS in severe obesity. ${ }^{117}$ After comparing the relationship of BMI-SDS to the waist circumferences, skinfolds, and fat mass data of 30,003 children, Freedman et al. concluded that BMI-SDS is weakly associated with other measures of adiposity in severe obesity and suggested expressing extreme BMIs relative to the 95th percentile or as a percentage of the 95th percentile, as opposed to using BMI-SDS. ${ }^{118}$ Despite this, BMI-SDS still continues to be widely used, including in recent publications. This is clearly apparent from the data available to us, which were almost entirely reported as BMI-SDS.

Previous literature has shown an increase in SBP, DBP, and BMI in older children. ${ }^{119}$ Our inclusion criteria specified ages from 4 to 19 years, and most of the studies analyzed covered a wide range of ages (Table 1). We did not have access to individual participants' data to evaluate the potential influence of individuals' ages at baseline on cardiovascular risk factors. However, data from four subsets of children up to 10 years ${ }^{50,64,64}$ did not suggest a different relationship from the whole cohort.

The exclusion of four data subsets for SBP, HDL, and LDL; and seven data subsets for TG before analysis $^{48,49,66,90,96}$ will likely have affected the outcome from the four meta-regression analyses and ultimately, 69 of the 71 studies reporting on cardiovascular outcomes were analyzed (Table 1). However, these subsets were impossible to compare due to the use of SBP-SDS, ${ }^{49,90}$ incorrect value for baseline TG IQR for the weight-loss subset ${ }^{48}$; lack of reported mean change in BMI-SDS for each subgroup in Kolsgaard et al. ${ }^{66}$; or inability to accurately determine SDs from the GMs of change in TG. ${ }^{96}$ Moreover, many studies $(69,78,58$, and 85 SBP, HDL, LDL, and TG data subsets, respectively) did not report the SD of mean change in BMI-SDS.

The original authors were contacted to retrieve values, although responses were limited. Imputation methods were required to estimate the $\mathrm{SD}$, based on the correlation between the absolute baseline and "end" value for SBP, HDL, LDL, and TG. However, this is not ideal, and authors should be encouraged to report both BMI-SDS change and $\mathrm{SD}$ - to facilitate future systematic reviews and meta- analyses. Finally, two outlier subsets ${ }^{76,109}$ were found to skew analyses for HDL and LDL.

Omitting the outlier Murer et al. ${ }^{76}$ in the LDL analysis had no effect on the minimum mean BMI-SDS required to improve LDL. Similarly, in the HDL analysis, there was no minimum BMI-SDS determinable that would lead to a beneficial increase in HDL, regardless of whether the control group in Visuthranukul et al. ${ }^{109}$ was omitted or not.

\section{Agreements and Disagreements with Other Studies}

Included in this review, a prospective cohort study by Ford et al. ${ }^{16}$ evaluating the impact of an outpatient weight management clinic in the United Kingdom on BMI-SDS in 88 adolescents with obesity found that significant clinical benefits were evident with a BMI-SDS reduction $\geq 0.5$, leading to a mean change in SBP, HDL, LDL, and TG from a baseline of $-3.5(-7.6$ to 0.7$), 0.09(95 \%-0.01$ to 0.18$)$, $-0.33(-0.53$ to -0.13$)$, and 0.70 (GM) $(0.59-0.84)$, respectively. These results differ after including all respective studies (minus outliers) in this review: suggesting that a minimum reduction in BMI-SDS of $1,1.2$, and 0.7 is required to ensure improvements in SBP, LDL, and TG; and that there was no discernible minimum change for HDL. This may be due to other confounding variables across the included 71 studies ( $n=69$ analyzed), which have been unaccounted for such as age, ethnicity, intensity and type of lifestyle intervention, and duration of followup; as well as the presence of alternative strong predictors of adult CVD, such as waist circumference and waist-toheight ratio (see clinical implications and recommendations for future research). Another prospective cohort study ( $n=230$; age range $7-17$ years) by Kolsgaard et al. reported a mean reduction of BMI-SDS of $-0.13(p<0.001)$ in their whole group after 1 year of intervention and linked this to a greater reduction in LDL. ${ }^{66}$ Participants with a decrease in BMI-SDS $\geq 0.23$ reduced their mean LDL and TG by 0.35 (SD $0.47 ; p<0.001)$ and $0.12(0.63 ; p=0.02)$, respectively, as opposed to $-0.1(0.49 ; p<0.001)$ and $-0.03(0.58 ; p=0.02)$, respectively, for individuals who had an increase in BMI-SDS at follow-up. However, no association was found between BMI-SDS reduction and improvement in HDL when comparing individuals with increased BMI-SDS and those with $a \geq 0.23$ reduction [mean change $0.02(0.23)$ vs. $-0.04(0.18) ; p=0.15]$. These findings are in keeping with the HDL, LDL, and TG analyses (Figs. 3-5) in this study; although this study was not included in this meta-regression, due to the lack of individual mean change in BMI-SDS reported for each of the four subgroups.

Our analysis in this review shows that BMI-SDS changes must be of a magnitude $(>0.7)$ rarely achieved in lifestyle intervention trials worldwide, to improve BP and lipid profile parameters. Previous meta-analyses have demonstrated a reduction in BMI-SDS after lifestyle intervention of -0.06 (95\% CI -0.12 to -0.01$)$ in children younger than 12 years and $-0.14(95 \%-0.18$ to -0.10$)$ in children 12 years and older at 6 months' follow-up. ${ }^{21} \mathrm{~A}$ 
meta-analysis of $33 \mathrm{RCTs}$ by Ho et al. illustrated a reduction of $-0.10(95 \% \mathrm{CI}-0.18$ to -0.02$)$ in BMI-SDS at 1 year from baseline with lifestyle interventions. ${ }^{22}$ Colquitt et al. found a mean reduction of -0.3 in BMI-SDS across 4 low-quality evidence RCTs, at 6-12 months of follow-up $(95 \% \mathrm{CI}-0.4$ to -0.2$){ }^{23}$

Recent meta-analyses by Al-Khudairy et al. and Mead et al. have shown that although lifestyle interventions in overweight and obese participants lead to significant reductions in BMI-SDS -0.34 (95\% CI -0.66 to -0.02$)$ in participants aged $12-17$ years; and $-0.06(95 \% \mathrm{CI}-0.10$ to -0.02$)$ in participants aged 6-11 years; they are unlikely to lead to clinical improvements in metabolic health due to inconsistent results, risk of bias, and low quality of evidence. ${ }^{120,121}$

To predict an improvement in adiposity in children and adolescents with obesity, we have suggested that future studies require a BMI-SDS reduction of at least $0.6^{31}$ Likewise, in this article, we suggest a magnitude of change in BMI-SDS seldom achieved in clinical practice, and these results challenge the efficacy of childhood weight management trials, as well as the validity of BMI-SDS as a measure of adiposity at extremes of obesity. Further, we have described significant improvements in insulin resistance and inflammation with a reduction in BMI-SDS $(p=0.003$ and 0.021 , respectively), although no minimum reduction in BMI-SDS could be identified to predict an improvement in metabolic biomarkers. ${ }^{32}$ These findings again highlight the importance of reporting additional indices of derived BMI values, which may better reflect changes in adiposity in children and adolescents with obesity.

\section{Clinical Implications}

Childhood obesity is a global health concern, with shortand long-term CVD risk implications, although there remains a paucity of data on the impact of BMI-SDS improvement through lifestyle modifications on cardio-metabolic risk. Setting a standard by which clinicians and researchers can assess the apparent success of their interventions should allow for the establishment of a clinically significant consensus on weight loss targets benefiting cardio-metabolic health in pediatrics, such as that seen in adult medical practice. ${ }^{13}$ However, the analyses in this review demonstrate that the required BMI-SDS reduction must be of a value seldom achieved in trials. It is uncertain whether the positive effects on SBP, LDL, HDL, and TG were attributable to weight loss, or rather to aspects of the lifestyle intervention independent of weight loss, such as a reduction in saturated fat intake or increased physical activity.

Furthermore, although BMI has been heralded as a strong predictor for childhood CVD risk factors by many large longitudinal and cross-sectional studies, ${ }^{122-124}$ it is likely that there are many other contributing factors to child and adolescent CVD. These include waist circumference and waist-to-height ratio, which have been described by Savva et al. in a study evaluating 1987 children (950 girls) with a mean age of $11.4 \pm 0.4$ years, as a better predictor of CVD risk factors in children than BMI. ${ }^{125}$
A cross-sectional study by Daniels et al., looking at one hundred ninety-two 7-17-year-old children, described that maturation stage, ethnicity, gender, and distribution of body fat should be interpreted with the results, if BMI is used as a measure of adiposity. ${ }^{126}$ This study took into consideration the length of follow-up and gender, but perhaps when incorporating BMI-SDS targets into future studies, the other variables that have been cited should be taken into consideration, to reduce confounding bias. This is especially the case when looking at all HDL data subsets in this review-where a minimum beneficial change in BMI-SDS could not be determined, due to the $95 \%$ PI including 0, even at extremes of BMISDS reduction.

\section{Recommendations for Future Research}

The current literature base suggests that very few childhood weight management trials to date are likely to achieve the BMI-SDS reduction targets, illustrated by this review, which would lead to beneficial changes in SBP, LDL, and TG. There are likely to be many other factors impacting the results found. Therefore, further large-scale reviews with sub-group analyses for treatment type; setting and intensity; age, using a cut-off of 10 years to divide children and adolescents into subgroups ${ }^{127}$; pubertal stage, according to Marshall and Tanner staging ${ }^{114}$; ethnicity; gender; duration of treatment and follow-up; family involvement; and waist circumference and waist-to-height ratio should be sought to improve the reliability of these findings; to elucidate the optimal duration, intensity, and style of intervention; and to allow for cost-effectiveness analysis. Further research of lifestyle interventions is also required in developing or under-developed countries.

Further, as with other reviews looking at lifestyle interventions and a reduction in BMI-SDS, heterogeneity is a major issue, in addition to the inconsistency of results and reporting bias. Thus, authors should be encouraged to report mean BMI-SDS, mean weight change, and SDs for these data, to facilitate future systematic reviews and meta-analyses.

\section{Conclusions}

The prevention of atherosclerotic CVD is particularly achieved by instituting and maintaining optimum health behaviors early in life. Using meta-regression, a BMI-SDS reduction of 1 is needed to ensure an improvement in SBP outcome in future clinical trials reporting on pediatric weight management outcomes. Likewise, a reduction of 1.2 and 0.7 is required in future clinical trials to ensure improvements in LDL and TG, respectively; although a reduction in BMI-SDS was significantly associated with an improvement in HDL, an equivalent value for HDL could not be determined. These figures should provide a benchmark for apparent success in future research, clinical practice, and the commissioning of weight management guidelines. 
Acknowledging the global burden of childhood obesity and the strong link between obesity and cardio-metabolic disease, dietary and physical activity professionals can use these results to judiciously support the cost-neutrality and effectiveness of their services as an integral component of public health and preventive cardiology. Further studies are needed to clarify the optimal duration, intensity, and setting for interventions - with cost-effectiveness analysis.

\section{Ethical Considerations}

All included studies have had their ethical approval taken into consideration before full analysis and inclusion.

\section{Authors' Contributions}

Dr. El-Medany managed the data, drafted and revised the article. Ms. Birch and Dr. Perry provided substantial contributions to the conception and design of the study; designed the data extraction instrument; performed electronic database searches, data screening, extraction, and quality assessment; coordinated and supervised data collection; and assisted with the drafting and revision of the article. Prof. Hamilton-Shield provided a substantial contribution to the conception and design of the study, conducted data screening and interpretation. Dr. Hunt provided statistical expertise in relation to study design, conducted the data analyses, and contributed to the drafting and revision of the article. Mr. Matson, Ms. Chong, and Ms. Beynon were involved in data acquisition and management. All authors approved the final article as submitted and agreed to be accountable for all aspects of the work.

\section{Acknowledgment}

The author would like to thank Dr. Suzanne Barr and the Preventive Cardiology faculty at Imperial College London for their support throughout the entire process.

\section{Funding Information}

This study was supported by the NIHR Biomedical Research Centre at University Hospitals Bristol NHS Foundation Trust and the University of Bristol. The views expressed in this publication are those of the authors and not necessarily those of the NHS, the National Institute for Health Research, or the Department of Health.

\section{Author Disclosure Statement}

Prof. Hamilton-Shield is a lead author on studies included in the systematic review that this article reports on. Dr. Hunt assisted with statistical analysis in Ford et al., ${ }^{13}$ which was included in the systematic review that this article reports on. The other authors have no conflicts of interest to disclose.

\section{Supplementary Material}

\author{
Supplementary Data S1 \\ Supplementary Data S2 \\ Supplementary Data S3 \\ Supplementary Figure S1 \\ Supplementary Table S1
}

\section{References}

1. World Health Organization. World health statistics 2016: monitoring health for the SDGs sustainable development goals. World Health Organization. June 8, 2016.

2. Pearce M, Webb-Phillips S, Bray I. Changes in objectively measured BMI in children aged 4-11 years: Data from the National Child Measurement Programme. J Public Health 2016;38: $459-466$.

3. Freedman DS, Dietz WH, Tang R, et al. The relation of obesity throughout life to carotid intima-media thickness in adulthood: The Bogalusa Heart Study. Int J Obes 2004;28:159.

4. Simmonds M, Llewellyn A, Owen CG, Woolacott N. Predicting adult obesity from childhood obesity: A systematic review and meta-analysis. Obes Rev 2016;17:95-107.

5. Pathobiological Determinants of Atherosclerosis in Youth (PDAY) Research Group. Relationship of atherosclerosis in young men to serum lipoprotein cholesterol concentrations and smoking: A preliminary report from the Pathobiological Determinants of Atherosclerosis in Youth (PDAY) Research Group. JAMA 1990;264:3018-3024.

6. Berenson GS, Srinivasan SR, Bao W, et al. Association between multiple cardiovascular risk factors and atherosclerosis in children and young adults. N Engl J Med 1998;338:1650-1656.

7. Li S, Chen W, Srinivasan SR, et al. Childhood cardiovascular risk factors and carotid vascular changes in adulthood: The Bogalusa Heart Study. JAMA 2003;290:2271-2276.

8. Magnussen CG, Venn A, Thomson R, et al. The association of pediatric low-and high-density lipoprotein cholesterol dyslipidemia classifications and change in dyslipidemia status with carotid intima-media thickness in adulthood: Evidence from the Cardiovascular Risk in Young Finns Study, the Bogalusa Heart Study, and the CDAH (Childhood Determinants of Adult Health) Study. $J$ Am Coll Cardiol 2009;53:860-869.

9. Lauer RM, Clarke WR, Beaglehole R. Level, trend, and variability of blood pressure during childhood: The Muscatine study. Circulation 1984;69:242-249.

10. Davis PH, Dawson JD, Riley WA, Lauer RM. Carotid intimalmedial thickness is related to cardiovascular risk factors measured from childhood through middle age: The Muscatine Study. Circulation 2001;104:2815-2819.

11. Raitakari OT, Juonala M, Rönnemaa T, et al. Cohort profile: The cardiovascular risk in Young Finns Study. Int J Epidemiol 2008; 37:1220-1226.

12. Magkos F, Fraterrigo G, Yoshino J, et al. Effects of moderate and subsequent progressive weight loss on metabolic function and adipose tissue biology in humans with obesity. Cell Metabolism 2016;23:591-601.

13. Jensen MD, Ryan DH, Apovian CM, et al. 2013 AHA/ACC/TOS guideline for the management of overweight and obesity in adults: A report of the American College of Cardiology/American Heart Association Task Force on Practice Guidelines and The Obesity Society. J Am Coll Cardiol 2014;63(25 Part B):2985-3023. 
14. Logue J, Thompson L, Romanes F, et al. Management of obesity: Summary of SIGN guideline. BMJ 2010;340:c154.

15. Reinehr T, Lass N, Toschke C, et al. Which amount of BMI-SDS reduction is necessary to improve cardiovascular risk factors in overweight children?. J Clin Endocrinol Metab 2016;101:31713179.

16. Ford AL, Hunt LP, Cooper A, Shield JP. What reduction in BMI SDS is required in obese adolescents to improve body composition and cardiometabolic health?. Arch Dis Child 2010;95:256-261.

17. Wunsch R, de Sousa G, Toschke AM, Reinehr T. Intima-media thickness in obese children before and after weight loss. Pediatrics 2006;118:2334-2340.

18. Cole TJ. The LMS method for constructing normalized growth standards. Eur J Clin Nutr 1990;44:45-60.

19. Brannsether B, Eide GE, Roelants M, et al. BMI and BMI SDS in childhood: Annual increments and conditional change. Ann Hum Biol 2017;44:28-33.

20. Poskitt EM. Defining childhood obesity: The relative body mass index (BMI). Acta Paediatr 1995;84:961-963.

21. Oude Luttikhuis H, Baur L, Jansen H, et al. Cochrane review: Interventions for treating obesity in children. Evidence-based child health. Cochrane Rev J 2009;4:1571-1729.

22. Ho M, Garnett SP, Baur L, et al. Effectiveness of lifestyle interventions in child obesity: Systematic review with metaanalysis. Pediatrics 2012;130:e1647-e1671.

23. Colquitt JL, Loveman E, O'Malley C, et al. Diet, physical activity, and behavioural interventions for the treatment of overweight or obesity in preschool children up to the age of 6 years. Cochrane Database Syst Rev 2016;10:CD012105.

24. Lobstein T, Baur L, Uauy R. Obesity in children and young people: A crisis in public health. Obes Rev 2004;5:4-85.

25. Hannon TS, Rao G, Arslanian SA. Childhood obesity and type 2 diabetes mellitus. Pediatrics 2005;116:473-480.

26. Urbina EM, Williams RV, Alpert BS, et al. Noninvasive assessment of subclinical atherosclerosis in children and adolescents: Recommendations for standard assessment for clinical research: A scientific statement from the American Heart Association. Hypertension 2009;54:919-950.

27. Pires A, Martins P, Pereira AM, et al. Insulin resistance, dyslipidemia and cardiovascular changes in a group of obese children. Arq Bras Cardiol 2014(AHEAD).

28. Cook S, Kavey RE. Dyslipidemia and pediatric obesity. Pediatr Clinics 2011;58:1363-1373.

29. Chiarelli F, Marcovecchio ML. Insulin resistance and obesity in childhood. Eur J Endocrinol 2008;159(suppl_1):S67-S74.

30. Tounian P, Aggoun Y, Dubern B, et al. Presence of increased stiffness of the common carotid artery and endothelial dysfunction in severely obese children: A prospective study. Lancet 2001; 358:1400-1404.

31. Birch L, Perry R, Hunt LP, et al. What change in body mass index is associated with improvement in percentage body fat in childhood obesity? A meta-regression. BMJ Open 2019;9:e028231.

32. Matson RI, Perry R, Hunt LP, et al. Change in obesity-related metabolic abnormalities associated with BMI improvement through life-style intervention: A meta-regression. Pediatr Diabetes 2020;21:173-193.

33. Moher D, Liberati A, Tetzlaff J, et al. Preferred reporting items for systematic reviews and meta-analyses: The PRISMA statement. 2009;89:873-880.

34. Birch L, Perry R, Penfold C, et al. What change in body mass index is needed to improve metabolic health status in childhood obesity: Protocol for a systematic review. Syst Rev 2016;5:120.
35. Higgins J, Green S. Cochrane Handbook for Systematic Reviews of Interventions. Chichester: Wiley, 2011.

36. Avenell A, Broom J, Brown TJ. Systematic review of the longterm outcomes of the treatments for obesity and implications for health improvement and the economic consequences for the National Health Service. Health Technol Assess 2004;4:iii-iv, $1-182$.

37. Harbord RM, Higgins JP. Meta-regression in Stata. Stata J 2008; $8: 493-519$.

38. Thompson SG, Higgins JP. How should meta-regression analyses be undertaken and interpreted?. Stat Med 2002;21:1559-1573.

39. Uysal Y, Wolters B, Knop C, Reinehr T. Components of the metabolic syndrome are negative predictors of weight loss in obese children with lifestyle intervention. Clin Nutr 2014;33: 620-625.

40. Reinehr T, de Sousa G, Wabitsch M. Changes of cardiovascular risk factors in obese children effects of inpatient and outpatient interventions. J Pediatr Gastroenterol Nutr 2006;43:506-511.

41. Bean MK, Mazzeo SE, Stern M, et al. Six-month dietary changes in ethnically diverse, obese adolescents participating in a multidisciplinary weight management program. Clin Pediatr 2011;50: 408-416.

42. Bell LM, Watts K, Siafarikas A, et al. Exercise alone reduces insulin resistance in obese children independently of changes in body composition. J Clin Endocrinol Metab 2007; 92:4230-4235.

43. Bock DE, Robinson T, Seabrook JA, et al. The Health Initiative Program for Kids (HIP Kids): Effects of a 1-year multidisciplinary lifestyle intervention on adiposity and quality of life in obese children and adolescents-a longitudinal pilot intervention study. BMC Pediatr 2014;14:296.

44. Boodai SA, McColl JH, Reilly JJ. National Adolescent Treatment Trial for Obesity in Kuwait (NATTO): Project design and results of a randomised controlled trial of a good practice approach to treatment of adolescent obesity in Kuwait. Trials 2014;15:234.

45. Bruyndonckx L, Hoymans VY, De Guchtenaere A, et al. Diet, exercise, and endothelial function in obese adolescents. Pediatrics 2015;135:e653-e661.

46. Bustos P, Orias J, Sáez K, et al. Effects of the Bright Bodies Program in Chilean obese children. Rev Med Chil 2015;143: $1136-1143$.

47. Calcaterra V, Larizza D, Codrons E, et al. Improved metabolic and cardiorespiratory fitness during a recreational training program in obese children. J Pediatr Endocrinol Metab 2013;26:271-276.

48. Corripio R, Gónzalez-Clemente JM, Pérez-Sánchez J, et al Weight loss in prepubertal obese children is associated with a decrease in adipocyte fatty-acid-binding protein without changes in lipocalin-2: A 2-year longitudinal study. Eur $J$ Endocrinol 2010;163:887-893.

49. Croker H, Viner RM, Nicholls D, et al. Family-based behavioural treatment of childhood obesity in a UK National Health Service setting: Randomized controlled trial. Int J Obes 2012; $36: 16$.

50. Dobe M, Geisler A, Hoffmann D, et al. The Obeldicks concept. An example for a successful outpatient lifestyle intervention for overweight or obese children and adolescents]. Bundesgesundheitsblatt Gesundheitsforschung Gesundheitsschutz 2011;54: $628-635$.

51. Doughty KN, Njike VY, Katz DL. Effects of a cognitivebehavioral therapy-based immersion obesity treatment program for adolescents on weight, fitness, and cardiovascular risk factors: A pilot study. Child Obes 2015;11:215-218. 
52. Evans RK, Franco RL, Stern M, et al. Evaluation of a 6-month multi-disciplinary healthy weight management program targeting urban, overweight adolescents: Effects on physical fitness, physical activity, and blood lipid profiles. Int $J$ Pediatr Obes 2009;4:130-133.

53. Farpour-Lambert NJ, Aggoun Y, Marchand LM, et al. Physical activity reduces systemic blood pressure and improves early markers of atherosclerosis in pre-pubertal obese children. $J \mathrm{Am}$ Coll Cardiol 2009;54:2396-2406.

54. Gajewska J, Kuryłowicz A, Mierzejewska E, et al. Complementary effects of genetic variations in LEPR on body composition and soluble leptin receptor concentration after 3-month lifestyle intervention in prepubertal obese children. Nutrients 2016;8:328.

55. Garanty-Bogacka B, Syrenicz M, Goral J, et al. Changes in inflammatory biomarkers after successful lifestyle intervention in obese children. Endokrynol Pol 2011;62:499-505.

56. Grønbæk H, Lange A, Birkebæk NH, et al. Effect of a 10-week weight loss camp on fatty liver disease and insulin sensitivity in obese Danish children. J Pediatr Gastroenterol Nutrit 2012;54: 223-228.

57. Kazankov K, Møller HJ, Lange A, et al. The macrophage activation marker sCD163 is associated with changes in NAFLD and metabolic profile during lifestyle intervention in obese children. Pediatr Obes 2015;10:226-233.

58. Grulich-Henn J, Lichtenstein S, Hörster F, et al. Moderate weight reduction in an outpatient obesity intervention program significantly reduces insulin resistance and risk factors for cardiovascular disease in severely obese adolescents. Int $J$ Endocrinol 2011;2011:541021.

59. Gunnarsdottir T, Einarsson SM, Njardvik U, et al. Family-based behavioral treatment for obese children-results and two year follow up. Laeknabladid 2014;100:139-145.

60. Huang F, del-Río-Navarro BE, de Castro GT, et al. Weight loss induced by 6 -month lifestyle intervention improves early endothelial activation and fibrinolysis in obese adolescents. Child Care Health Dev 2011;37:377-384

61. Huang F, del-Río-Navarro BE, Pérez-Ontiveros JA, et al. Effect of six-month lifestyle intervention on adiponectin, resistin and soluble tumor necrosis factor- $\alpha$ receptors in obese adolescents. Endocr J 2014;61:921-931.

62. Hvidt KN, Olsen MH, Holm JC, Ibsen H. Obese children and adolescents have elevated nighttime blood pressure independent of insulin resistance and arterial stiffness. Am J Hypertens 2014; 27:1408-1415.

63. Kalavainen M, Utriainen P, Vanninen E, et al. Impact of childhood obesity treatment on body composition and metabolic profile. World J Pediatr 2012;8:31-37.

64. Kirk S, Zeller M, Claytor R, et al. The relationship of health outcomes to improvement in BMI in children and adolescents. Obes Res 2005; 13:876-882.

65. Kolotourou M, Radley D, Chadwick P, et al. Is BMI alone a sufficient outcome to evaluate interventions for child obesity?. Child Obes 2013;9:350-356.

66. Kolsgaard ML, Joner G, Brunborg C, et al. Reduction in BMI Z-score and improvement in cardiometabolic risk factors in obese children and adolescents. The Oslo Adiposity Intervention Study - A hospital/public health nurse combined treatment. BMC Pediatr 2011;11:47.

67. Mager DR, Iñiguez IR, Gilmour S, Yap J. The effect of a low fructose and low glycemic index/load (FRAGILE) dietary intervention on indices of liver function, cardiometabolic risk factors, and body composition in children and adolescents with nonal- coholic fatty liver disease (NAFLD). J Parenter Enteral Nutr 2015;39:73-84.

68. Makkes S, Renders CM, Bosmans JE, et al. One-year effects of two intensive inpatient treatments for severely obese children and adolescents. BMC Pediatr 2016;16:120.

69. Marcano H, Fernández M, Paoli M, et al. Limited weight loss or simply no weight gain following lifestyle-only intervention tends to redistribute body fat, to decrease lipid concentrations, and to improve parameters of insulin sensitivity in obese children. Int $J$ Pediatr Endocrinol 2011;2011:241703.

70. Meyer AA, Kundt G, Lenschow U, et al. Improvement of early vascular changes and cardiovascular risk factors in obese children after a six-month exercise program. $J$ Am Coll Cardiol 2006;48: $1865-1870$.

71. Miraglia F, de Moraes Silveira CR, Gomes Beghetto M, et al Behavior of adipokines after a year follow-up in the obesity outpatient clinic for children and adolescents. Nutr Hosp 2015;32: $1554-1559$.

72. Montero D, Walther G, Perez-Martin A, et al. Effects of a lifestyle program on vascular reactivity in macro-and microcirculation in severely obese adolescents. J Clin Endocrinol Metab 2014;99:1019-1026.

73. Morell-Azanza L, García-Calzón S, Rendo-Urteaga T, et al Serum oxidized low-density lipoprotein levels are related to cardiometabolic risk and decreased after a weight loss treatment in obese children and adolescents. Pediatr Diabetes 2017;18:392-398.

74. Rendo-Urteaga T, García-Calzón S, González-Muniesa P, et al Peripheral blood mononuclear cell gene expression profile in obese boys who followed a moderate energy-restricted diet: Differences between high and low responders at baseline and after the intervention. Br J Nutr 2015;113:331-342.

75. Murdolo G, Tortoioli C, Celi F, et al. Fetuin-A, adiposity-linked insulin resistance and responsiveness to an educational-based weight excess reduction program: A population-based survey in prepubertal schoolchildren. Endocrine 2017;56:357-365.

76. Murer SB, Knöpfli BH, Aeberli I, et al. Baseline leptin and leptin reduction predict improvements in metabolic variables and longterm fat loss in obese children and adolescents: A prospective study of an inpatient weight-loss program. Am J Clin Nutr 2011; 93:695-702.

77. Aeberli I, Jung A, Murer SB, et al. During rapid weight loss in obese children, reductions in TSH predict improvements in insulin sensitivity independent of changes in body weight or fat. $J$ Clin Endocrinol Metab 2010;95:5412-5418.

78. Obert $\mathrm{P}$, Gueugnon $\mathrm{C}$, Nottin $\mathrm{S}$, et al. Impact of diet and exercise training-induced weight loss on myocardial mechanics in severely obese adolescents. Obesity 2013;21:2091-2098.

79. Pacifico L, Arca M, Anania C, et al. Arterial function and structure after a 1-year lifestyle intervention in children with nonalcoholic fatty liver disease. Nutr Metab Cardiovasc Dis 2013;23:1010-1016.

80. Parillo M, Licenziati MR, Vacca M, et al. Metabolic changes after a hypocaloric, low-glycemic-index diet in obese children. $J$ Endocrinol Invest 2012;35:629-633.

81. Racil G, Ounis OB, Hammouda O, et al. Effects of high vs. moderate exercise intensity during interval training on lipids and adiponectin levels in obese young females. Eur J Appl Physiol 2013;113:2531-2540.

82. Racil G, Coquart JB, Elmontassar W, et al. Greater effects of high-compared with moderate-intensity interval training on cardio-metabolic variables, blood leptin concentration and ratings of perceived exertion in obese adolescent females. Biol Sport 2016;33:145 
83. Rambhojan C, Bouaziz-Amar E, Larifla L, et al. Ghrelin, adipokines, metabolic factors in relation with weight status in school-children and results of a 1-year lifestyle intervention program. Nutr Metab 2015;12:43.

84. Reinehr T, Andler W. Changes in the atherogenic risk factor profile according to degree of weight loss. Arch Dis Child 2004; $89: 419-422$

85. Reinehr T, de Sousa G, Toschke AM, Andler W. Long-term follow-up of cardiovascular disease risk factors in children after an obesity intervention. Am J Clin Nutr 2006;84:490-496.

86. Reinehr T, Roth CL. Fetuin-A and its relation to metabolic syndrome and fatty liver disease in obese children before and after weight loss. J Clin Endocrinol Metab 2008;93:4479-4485.

87. Reinehr T, Stoffel-Wagner B, Roth CL. Retinol-binding protein 4 and its relation to insulin resistance in obese children before and after weight loss. J Clin Endocrinol Metab 2008;93:2287-2293.

88. Reinehr T, Kleber M, Toschke AM. Lifestyle intervention in obese children is associated with a decrease of the metabolic syndrome prevalence. Atherosclerosis 2009;207:174-180.

89. Reinehr T, Elfers C, Lass N, Roth CL. Irisin and its relation to insulin resistance and puberty in obese children: A longitudinal analysis. J Clin Endocrinol Metab 2015;100:2123-2130.

90. Rijks JM, Plat J, Mensink RP, et al. Children with morbid obesity benefit equally as children with overweight and obesity from an ongoing care program. J Clin Endocrinol Metab 2015;100:35723580 .

91. Rohrer TR, Rizzo VF, Cäsar JJ, et al. Changes in hepatic risk factors, metabolic variables, body composition, and physical fitness in obese children after a one-year weight loss program. J Pediatr Endocrinol Metab 2008;21:837-846.

92. Roth CL, Kratz M, Ralston MM, Reinehr T. Changes in adiposederived inflammatory cytokines and chemokines after successful lifestyle intervention in obese children. Metabolism 2011;60:445452.

93. Roth CL, Elfers C, Lass N, Reinehr T. Betatrophin: No relation to glucose metabolism or weight status in obese children before and after lifestyle intervention. Pediatr Diabetes 2017;18:485-491.

94. Rovira FA, Miro PN, Zaragoza-Jordana M, et al. Clinical and metabolic effectiveness of a new motivational therapy for the treatment of obesity in adolescents (OBEMAT). An Pediatr 2013; 78:157-166

95. Santomauro M, Paoli-Valeri $M$, Fernández $M$, et al. Nonalcoholic fatty liver disease and its association with clinical and biochemical variables in obese children and adolescents: Effect of a one-year intervention on lifestyle. Endocrinol Nutr 2012;59: 346-353.

96. Savoye M, Nowicka P, Shaw M, et al. Long-term results of an obesity program in an ethnically diverse pediatric population. Pediatrics 2011;127:402.

97. Savoye M, Shaw M, Dziura J, et al. Effects of a weight management program on body composition and metabolic parameters in overweight children: A randomized controlled trial. JAMA 2007;297:2697-2704.

98. Savoye M, Caprio S, Dziura J, et al. Reversal of early abnormalities in glucose metabolism in obese youth: Results of an intensive lifestyle randomized controlled trial. Diabetes care 2014;37:317-324.

99. Seabra A, Katzmarzyk P, Carvalho MJ, et al. Effects of 6-month soccer and traditional physical activity programmes on body composition, cardiometabolic risk factors, inflammatory, oxidative stress markers and cardiorespiratory fitness in obese boys. Journal of sports sciences 2016;34:1822-1829.
100. Shalitin S, Ashkenazi-Hoffnung L, Yackobovitch-Gavan M, et al. Effects of a twelve-week randomized intervention of exercise and/or diet on weight loss and weight maintenance, and other metabolic parameters in obese preadolescent children. Horm Res Paediatr 2009;72:287-301.

101. Tan-Ting AM, Llido L. Outcome of a hospital based multidisciplinary weight loss program in obese Filipino children. Nutrition 2011;27:50-54.

102. Truby H, Baxter $\mathrm{K}$, Ware RS, et al. A randomized controlled trial of two different macronutrient profiles on weight, body composition and metabolic parameters in obese adolescents seeking weight loss. PLoS One 2016;11:e0151787.

103. Jiménez MV, Martos R, Morales RM, et al. Relationship between changes in plasma leptin concentrations and plasminogen activator inhibitor- 1 in obese prepubertal children after nine months of treatment. Ann Nutr Metab 2013;63:216-222.

104. van der Baan-Slootweg O, Benninga MA, Beelen A, et al. Inpatient treatment of children and adolescents with severe obesity in the Netherlands: A randomized clinical trial. JAMA Pediatr 2014; $168: 807-814$

105. Van Hoorenbeeck K, Franckx H, Debode P, et al. Metabolic disregulation in obese adolescents with sleep-disordered breathing before and after weight loss. Obesity 2013;21:1446-1450.

106. Verduci E, Radaelli G, Salvioni M, et al. Plasma long-chain fatty acids profile and metabolic outcomes in normolipidaemic obese children after one-year nutritional intervention. Acta Paediatr 2011;100:585-589.

107. Pozzato C, Verduci E, Scaglioni S, et al. Liver fat change in obese children after a 1-year nutrition-behavior intervention. $J$ Pediatr Gastroenterol Nutr 2010;51:331-335.

108. Verduci E, Lassandro C, Giacchero R, et al. Change in metabolic profile after 1-year nutritional-behavioral intervention in obese children. Nutrients 2015;7:10089-10099.

109. Visuthranukul C, Sirimongkol P, Prachansuwan A, et al. Lowglycemic index diet may imposrove insulin sensitivity in obese children. Pediatr Res 2015;78:567.

110. Vitola BE, Deivanayagam S, Stein RI, et al. Weight loss reduces liver fat and improves hepatic and skeletal muscle insulin sensitivity in obese adolescents. Obesity 2009;17:1744-1748.

111. Vos RC, Wit JM, Pijl H, Houdijk EC. Long-term effect of lifestyle intervention on adiposity, metabolic parameters, inflammation and physical fitness in obese children: A randomized controlled trial. Nutr Diabetes 2011;1:e9

112. Weigel C, Kokocinski K, Lederer P, et al. Childhood obesity: Concept, feasibility, and interim results of a local group-based, long-term treatment program. J Nutr Educ Behav 2008;40:369-373.

113. Weiss R, Shaw M, Savoye M, Caprio S. Obesity dynamics and cardiovascular risk factor stability in obese adolescents. Pediatr Diabetes 2009;10:360-367.

114. Tanner JM. Growth at adolescence: With a general consideration of the effects of hereditary and environmental factors upon growth and maturation from birth to maturity. 2nd edition. 1962.

115. Haney EM, Huffman LH, Bougatsos C, et al. Screening and treatment for lipid disorders in children and adolescents: Systematic evidence review for the US Preventive Services Task Force. Pediatrics 2007;120:e189-e214.

116. Vanderwall C, Eickhoff J, Clark RR, Carrel AL. BMI z-score in obese children is a poor predictor of adiposity changes over time. BMC Pediatr 2018;18:187.

117. Júlíusson PB, Roelants M, Benestad B, et al. Severe obesity is a limitation for the use of body mass index standard deviation scores in children and adolescents. Acta Paediatr 2018;107:307-314. 
118. Freedman DS, Butte NF, Taveras EM, et al. BMI z-scores are a poor indicator of adiposity among 2-to 19-year-olds with very high BMIs, NHANES 1999-2000 to 2013-2014. Obesity 2017; 25:739-746.

119. Fraporti MI, Adami FS, Rosolen MD. Cardiovascular risk factors in children. Rev Port Cardiol 2017;36:699-70.

120. Al-Khudairy L, Loveman E, Colquitt JL, et al. Diet, physical activity and behavioural interventions for the treatment of overweight or obese adolescents aged 12 to 17 years. Cochrane Database Sys Rev 2017;6:CD012691.

121. Mead E, Brown T, Rees K, et al. Diet, physical activity and behavioural interventions for the treatment of overweight or obese children from the age of 6 to 11 years. Cochrane Database Syst Rev 2017;6:CD012651.

122. Freedman DS, Khan LK, Serdula MK, et al. The relation of childhood BMI to adult adiposity: The Bogalusa Heart Study. Pediatrics 2005;115:22-27.

123. Baker JL, Olsen LW, Sørensen TI. Childhood body-mass index and the risk of coronary heart disease in adulthood. $N$ Engl J Med 2007;357:2329-2337.

124. Burke V, Beilin LJ, Simmer K, et al. Predictors of body mass index and associations with cardiovascular risk factors in Australian children: A prospective cohort study. Int J Obes 2005;29:15.

125. Savva SC, Tornaritis M, Savva ME, et al. Waist circumference and waist-to-height ratio are better predictors of cardiovascular disease risk factors in children than body mass index. Int $J$ Obes 2000;24:1453.

126. Daniels SR, Khoury PR, Morrison JA. The utility of body mass index as a measure of body fatness in children and adolescents: Differences by race and gender. Pediatrics 1997;99:804-807.

\section{Appendix A1. Eligibility Criteria for Inclusion to the Systematic}

\section{Review}

\section{Participants}

Studies with participants aged 4, up to 19 years, with a diagnosis of obesity by using defined BMI thresholds and centiles, were considered for inclusion. BMI-standard deviation score (SDS) was calculated as a function of the degree of obesity of the subjects when compared with BMI standards; including, but not limited to: the 98th BMI centile on the UK 1990 growth reference chart, ${ }^{\mathrm{A} 1}$ 95th $\mathrm{BMI}$ percentile on the US Centre for Disease Control and Prevention growth chart, ${ }^{\mathrm{A} 2}$ the International Obesity Taskforce (IOTF) BMI for age cut-off points, ${ }^{\mathrm{A} 3}$ and the WHO growth references, ${ }^{\mathrm{A} 4, \mathrm{~A} 5}$ in addition to country-specific obesity thresholds, using BMI reference data from their pediatric populations.

Overweight individuals, pregnant women, or those with a critical illness, endocrine disorder, or syndromic obesity were excluded from this review.
127. Sacks D, Canadian Paediatric Society, Adolescent Health Committee. Age limits and adolescents. Paediatr Child Health 2003; 8:577-578.

128. Cole TJ, Freeman JV, Preece MA. Body mass index reference curves for the UK, 1990. Arch Dis Child 1995;73:25-29.

129. Kuczmarski RJ. CDC growth charts: United States. US Department of Health and Human Services, Centers for Disease Control and Prevention, National Center for Health Statistics, 2000.

130. Cole TJ, Lobstein T. Extended international (IOTF) body mass index cut-offs for thinness, overweight and obesity. Pediatr Obes 2012;7:284-294.

131. De Onis M, Garza C, Onyango AW, Borghi E. Comparison of the WHO child growth standards and the CDC 2000 growth charts. $J$ Nutr 2007;137:144-148.

132. Onis MD, Onyango AW, Borghi E, et al. Development of a WHO growth reference for school-aged children and adolescents. Bull World Health Organ 2007;85:660-667.

Address correspondence to: Ahmed Y.M. El-Medany, MSc Cardiology Department Bristol Royal Infirmary University Hospitals Bristol NHS Foundation Trust Marlborough Street Bristol BS2 8HW United Kingdom

E-mail: amedany@doctors.org.uk

\section{Interventions}

Studies of lifestyle interventions that involved dietary, exercise, and/or behavioral components, with the aim of reducing obesity, were included. Interventions less than 2 weeks in duration and those that involved surgical and/or pharmacological components (e.g., bariatric surgery, drug therapy) were excluded. Studies focusing on obesity prevention were also excluded. No restrictions were imposed regarding the intervention setting or delivery.

\section{Outcome Measures}

To meet the inclusion criteria, interventions had to report baseline (pre-) and post-intervention BMI-SDS or change measurements of BMI-SDS plus one or more of the following markers of metabolic health:

- Adiposity measures other than BMI (including waist circumference and percentage body fat)

- Glucose

- Insulin sensitivity/resistance (homeostatic model assessment)

- Lipid profile (triglycerides, total cholesterol, low-density lipoprotein [LDL]/high-density lipoprotein [HDL] cholesterol) 

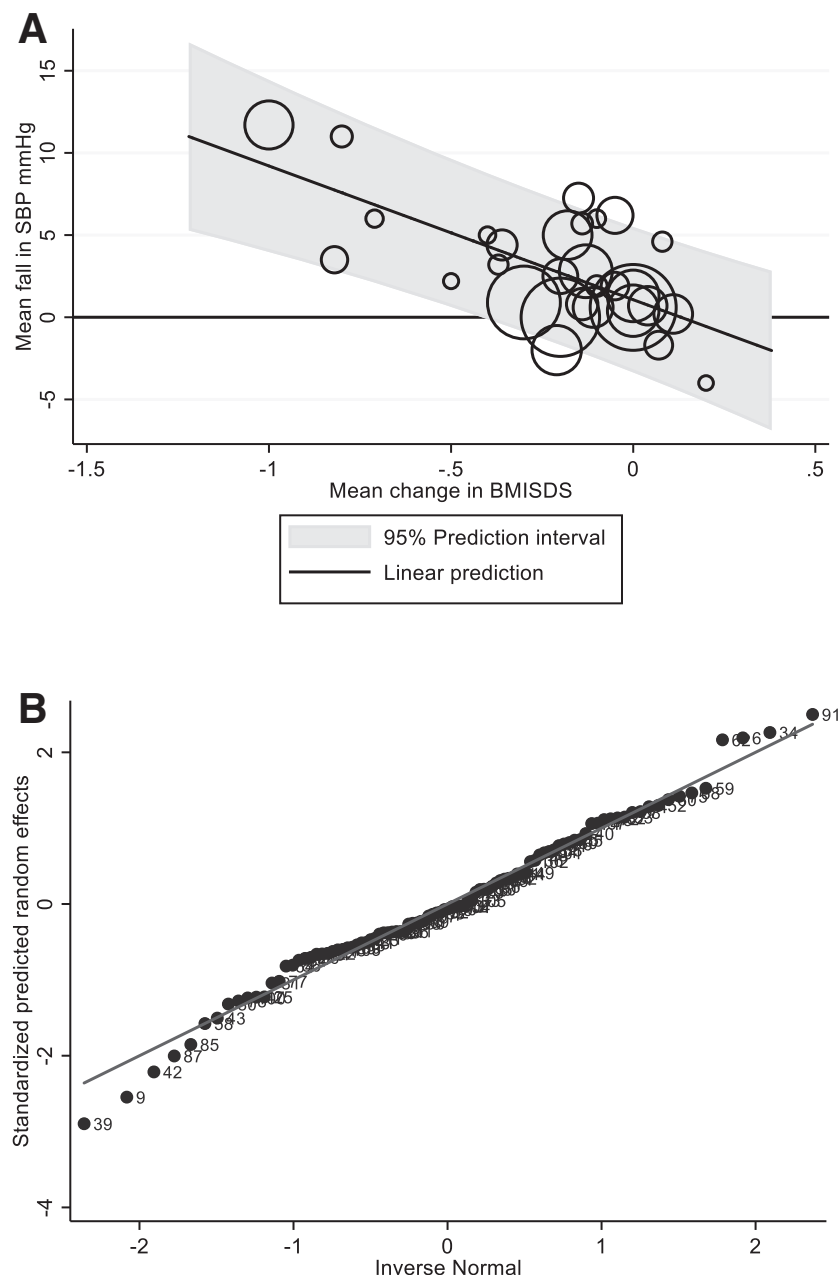

Appendix Figure A1. (A) Meta-regression line showing the relationship between mean change in BMI-SDS and SBP $(n=30)$. (B) Half-normal plot of the standardized predicted random effects from the SBP meta-regression $(n=99)$. SBP, systolic blood pressure; SDS, standard deviation score.

- Inflammation (C-reactive protein)

- Blood pressure (systolic, diastolic)

- Liver function

This article focuses on blood pressure and lipid profile only. The other two papers in this series report on the remaining outcome measures.

\section{Study Design}

Completed, published, randomized controlled trials (RCTs) and nonrandomized studies (cohort studies) of lifestyle interventions, with or without follow-up.

\section{Report Characteristics}

No language restrictions were imposed. Articles were translated as required by using the University of Bristol translation services. No publication date restrictions were imposed.
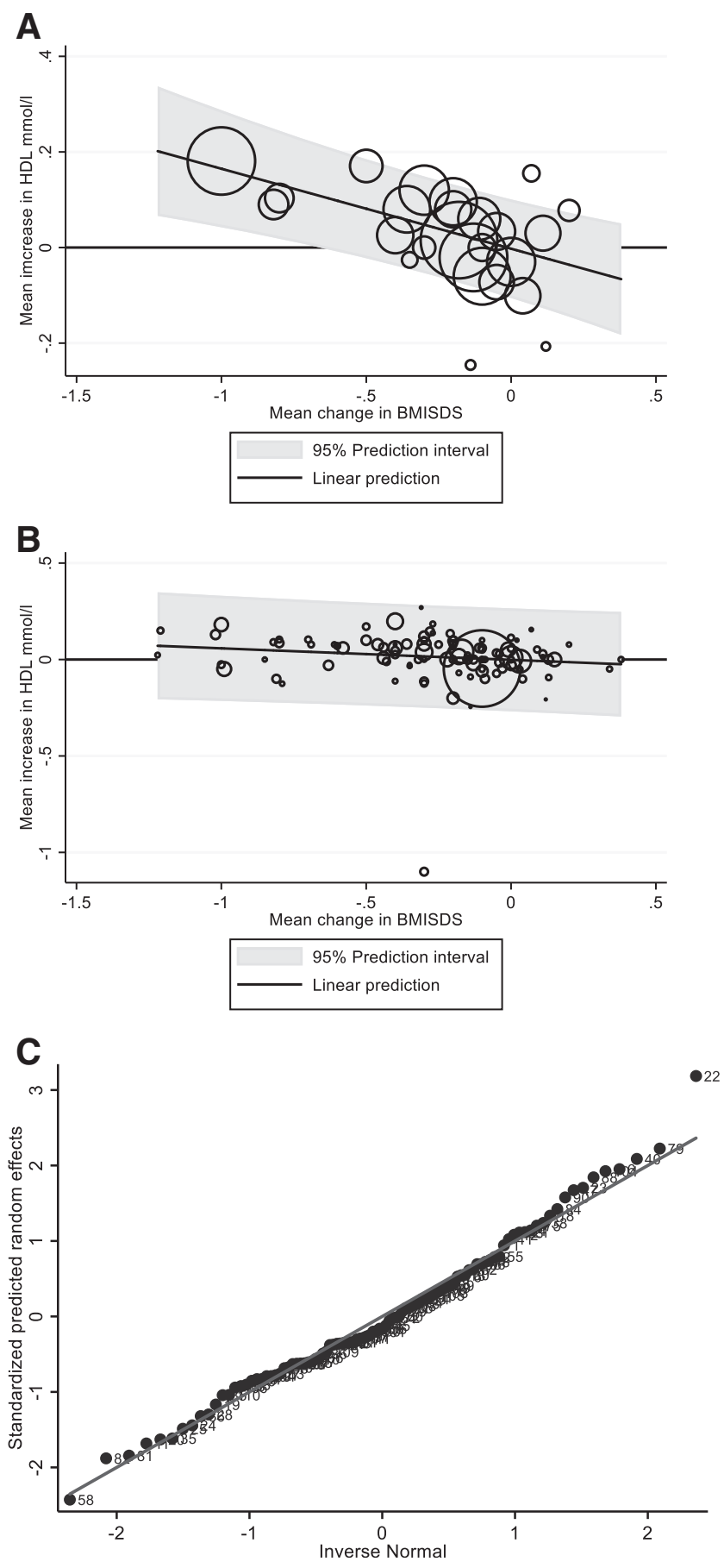

Appendix Figure A2. (A) Meta-regression line showing the relationship between mean change in BMI-SDS and HDL $(n=25)$. (B) Meta-regression line showing the relationship between mean change in BMI-SDS and HDL, including an outlying observation $(n=104)$. (C) Half-normal plot of the standardized predicted random effects from the HDL meta-regression, omitting an outlying observation $(n=103)$. HDL, high-density lipoprotein.

\section{Information Sources and Search Methods}

Studies were identified by searching electronic databases, scanning reference lists of articles, and through li- 

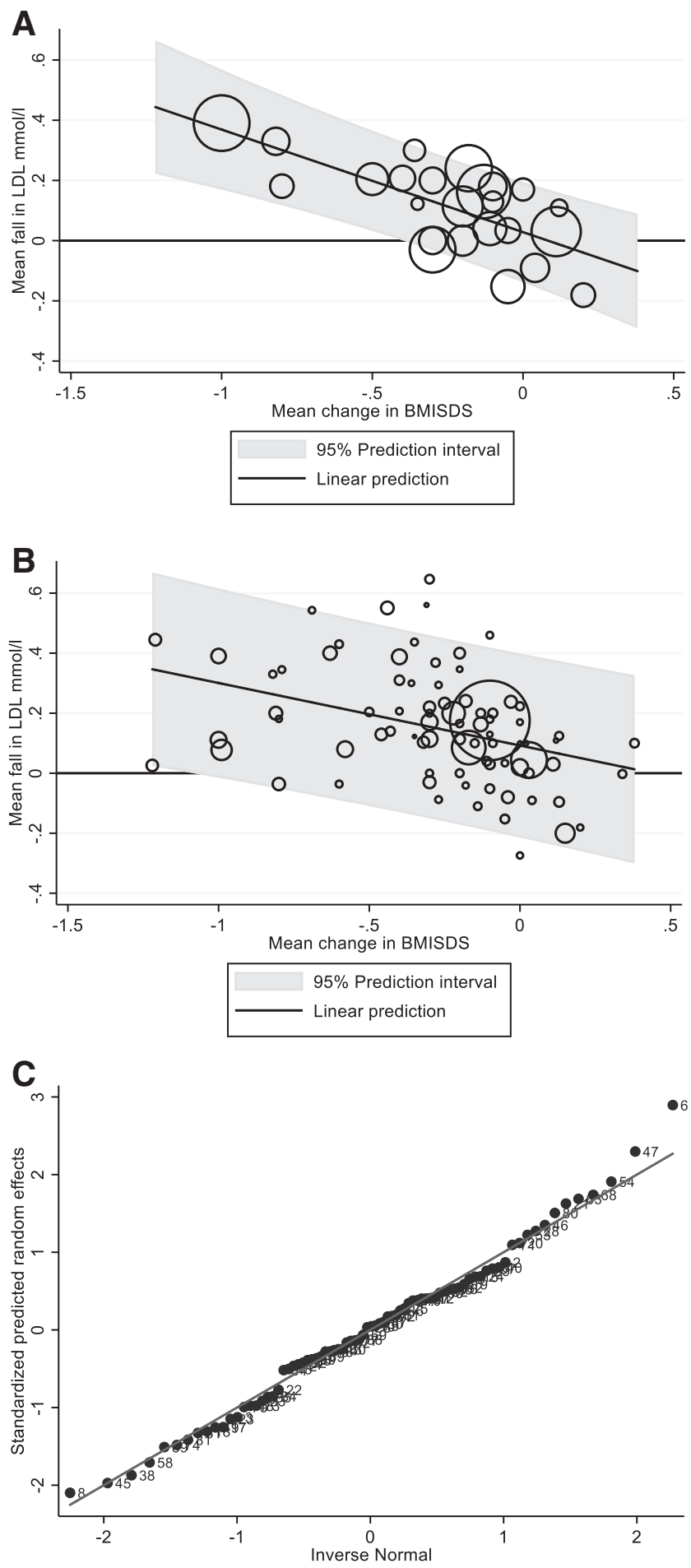

Appendix Figure A3. (A) Meta-regression line showing the relationship between mean change in BMI-SDS and LDL $(n=24)$. (B) Meta-regression line showing the relationship between mean change in BMI-SDS and LDL, omitting an outlying observation $(n=81)$. (C) Half-normal plot of the standardized predicted random effects from the LDL meta-regression, omitting the outlying observation $(n=81)$. LDL, low-density lipoprotein.
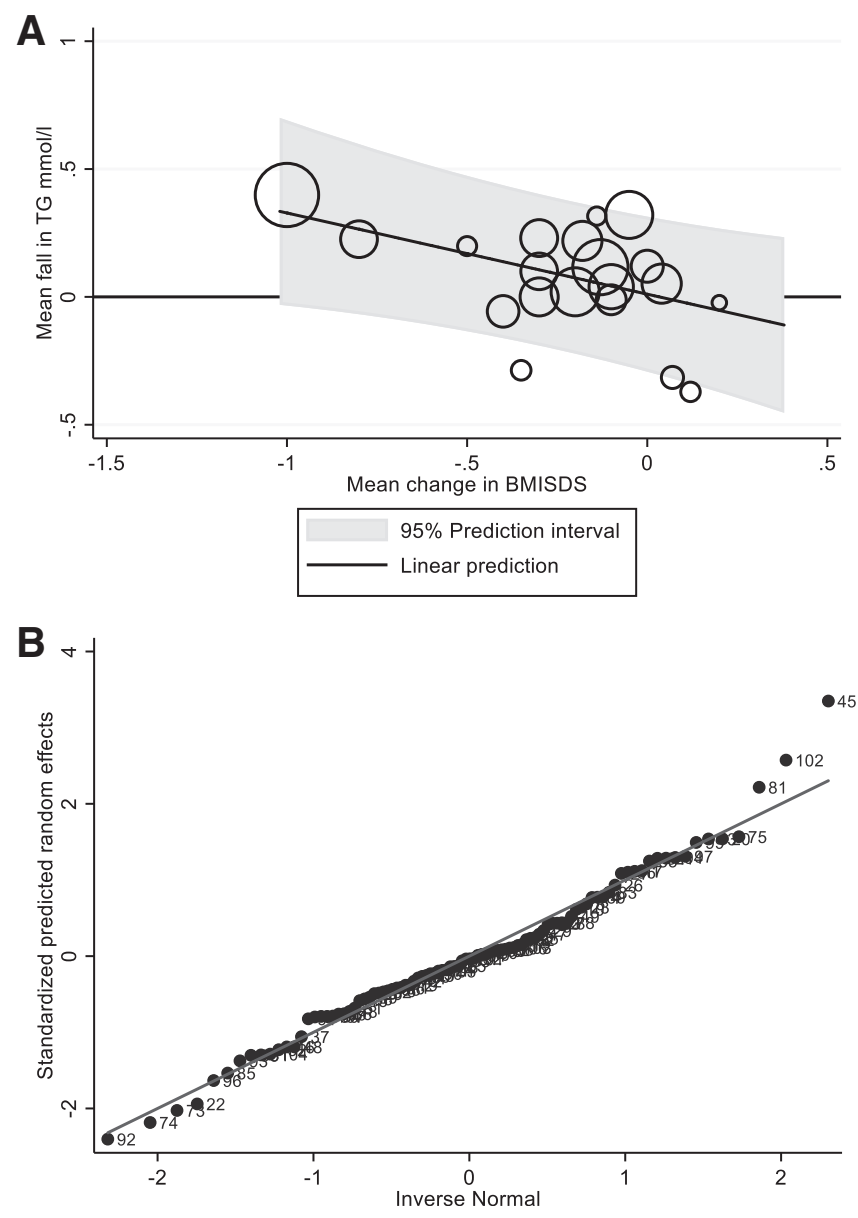

Appendix Figure A4. (A) Meta-regression line showing the relationship between mean change in BMI-SDS and TG $(n=20)$. (B) Half-normal plot to show the standardized predicted random effects from the TG meta-regression $(n=98)$. TG, triglyceride.

aison with relevant experts as appropriate. Literature search strategies were developed by using a combination of Medical Subject Headings (MeSH) and keyword terms.

The following electronic databases were searched from their inception: AMED (from 1985), Embase (from 1974), and MEDLINE (from 1946) all via OVID, Web of Science, and CENTRAL via Cochrane library. Search strategies were adapted appropriately for each database to accommodate differences in controlled vocabulary and syntax rules. Conference abstracts were not included in this review but were used to identify any potential studies and to contact authors regarding the availability of full-text articles.

\section{Study Selection}

Titles and abstracts were assessed for suitability by two independent reviewers from the review team. Articles that appeared to meet the inclusion criteria were retrieved in 
full and independently screened for inclusion by two reviewers. Disagreements in opinion regarding the studies considered for inclusion were resolved through discussion, and the reasons for excluding studies were recorded. Reference lists of included studies were hand-searched for additional articles, and the full-text articles of relevant studies identified were retrieved and reviewed for inclusion by two reviewers.

\section{Data Management}

The EndNote reference management software package was used to manage all the search results throughout the review period. Literature search results were imported into an EndNote library, and duplicates were removed.

\section{Data Collection}

Data were extracted from full-text articles identified for inclusion independently by two reviewers from the review team, by using a standardized data extraction form. The extraction form was piloted before starting the review and refined accordingly to ensure consistency. Disagreements were resolved through discussion between the reviewers.

Information was extracted from each included study on:

- Study design (RCT/cohort)

- Definition of obesity

- Characteristics of study participants (including number of participants, age, sex, pubertal status, ethnicity, and socioeconomic status) and the study's inclusion and exclusion criteria

- Intervention (including location, content, format, duration, delivery)

- Dietary quality (Mediterranean, low glycemic index, "traffic-light," calorie-restricted diet)

- Metabolic parameters measured (see the Outcome Measures section above)

- Results

- Analysis methods

- Study limitations

\section{Appendix A2. Analysis Results}

Appendix Figure A1(A) shows the results of the analysis and the fitted regression line for the 30 subsets that explicitly reported standard deviation (SDs) associated with the change in SBP. The adjusted $R^{2}$ was $53 \%$. There was, however, significant between-study heterogeneity, and the $I^{2}$ was $70 \%$.

The equation for the fitted line was: Mean change in $\mathrm{SBP}=-8.139 \times$ Mean change in BMI-SDS +1.067 . It was estimated that a future dataset with a mean reduction of BMI-SDS of 0.43 or more would have a $95 \%$ prediction interval $(95 \% \mathrm{PI})$ that excludes 0 , that is, would be compatible with a mean reduction of SBP; however, relatively few subsets had changes of this magnitude.

A half-normal plot for the standardized predicted random effects is shown in Appendix Fig. A1(B). Most were within \pm 2 compatible with a normal distribution; although it should be noted that the datasets themselves were not wholly independent, as some came from the same studies.

Appendix Figure A2(A) shows no significant relationship between mean change in BMI-SDS and mean change in HDL among the 25 subsets that reported SDs for the latter. The equation of the fitted line was: Mean change (increase) in $\mathrm{HDL}=-0.167 \times$ Mean change in BMI-SDS -0.0025 , with the $95 \%$ PI suggesting that a reduction of the mean BMI-SDS by at least 0.69 should ensure an increase in mean HDL (adjusted $R^{2}=56 \%$; $\left.I^{2}=65 \%\right)$.

The analysis in Appendix Figure A2(B) is very skewed by the outlier control subset in Visuthranukul et al. ${ }^{\mathrm{A} 6}$ (the mean change in HDL for this subset was -1.1). The equation of the fitted line was: Mean change (increase) in $\mathrm{HDL}=-0.060 \times$ Mean change in BMI-SDS -0.002 . The slope was not statistically significant ( $p=0.168 ; 95 \% \mathrm{CI}$ -0.145 to 0.026 ) and shows that the BMI-SDS needed to ensure that an improvement in mean HDL was beyond the range of the data (in excess of 1.22) (adjusted $R^{2}=1 \%$; $\left.I^{2}=94 \%\right)$.

A half-normal plot for the standardized predicted random effects is shown in Appendix Figure A2(C). Most were within \pm 2 , expected from a normal distribution.

Analyses of the 24 subsets that reported SDs for the changes in LDL are shown in Appendix Figure A3(A). The equation of the fitted line was: Mean change in LDL $=$ $-0.341 \times$ mean change in mean BMI-SDS +0.028 (adjusted $R^{2}=65 \% ; I^{2}=44 \%$ ). The $95 \%$ PI suggested that a mean BMI-SDS reduction of 0.4 might ensure a reduction in mean LDL; however, a few had changes of this magnitude and this result did not seem to be corroborated by the analysis using all 82 data subsets (see main text and Fig. 4).

Appendix Figure A3(B) shows the meta-regression line fitted in Figure 4 of the main text but omitting the outlier Murer et al. ${ }^{\mathrm{A} 7}$ The equation of the line was: Mean change in $\mathrm{LDL}=-0.208 \times$ mean change in mean BMI-SDS +0.092 (adjusted $R^{2}=16 \% ; I^{2}=86 \%$ ). This analysis still suggested that a reduction of at least 1.2 in mean BMI-SDS would be needed to ensure a reduction in mean LDL.

A half-normal plot for the standardized predicted random effects is shown in Appendix Figure A3(C). Most effects were within \pm 2 , as expected from a normal distribution.

Appendix Figure A4(A) shows the fitted regression line for the 20 subgroups that reported SDs for mean change in triglyceride (TG) (adjusted $R^{2}=32 \% ; I^{2}=70 \%$.) The equation of the fitted line was: mean fall in $\mathrm{TG}=-0.318 \times$ mean change in BMI-SDS +0.011 . From the $95 \%$ PI shown, reductions of less than 1 in the mean BMI-SDS would be unlikely to ensure a mean fall in TG in any future study.

A half-normal plot for the standardized predicted random effects is shown in Appendix Figure A4(B). Most effects were within the \pm 2 expected from a normal distribution.

(Appendices continue $\rightarrow$ ) 


\section{Appendix References}

A1. Cole TJ, Freeman JV, Preece MA. Body mass index reference curves for the UK, 1990. Arch Dis Child 1995;73:25-29.

A2. Kuczmarski RJ. CDC growth charts; United States. 2000.

A3. Cole TJ, Lobstein T. Extended international (IOTF) body mass index cut-offs for thinness, overweight and obesity. Pediatr Obes 2012;7:284-294.

A4. De Onis M, Garza C, Onyango AW, Borghi E. Comparison of the WHO child growth standards and the CDC 2000 growth charts. $J$ Nutr 2007;137:144-148.
A5. Onis MD, Onyango AW, Borghi E, et al. Development of a WHO growth reference for school-aged children and adolescents. Bull World Health Organ 2007;85:660-667.

A6. Visuthranukul C, Sirimongkol P, Prachansuwan A, et al. Lowglycemic index diet may imposrove insulin sensitivity in obese children. Pediatr Res 2015;78:567.

A7. Murer SB, Knöpfli BH, Aeberli I, et al. Baseline leptin and leptin reduction predict improvements in metabolic variables and longterm fat loss in obese children and adolescents: A prospective study of an inpatient weight-loss program. Am J Clin Nutr 2011; 93:695-702. 